\title{
A climatology of polar stratospheric cloud composition between 2002 and 2012 based on MIPAS/Envisat observations
}

\author{
Reinhold Spang $^{1}$, Lars Hoffmann ${ }^{2}$, Rolf Müller ${ }^{1}$, Jens-Uwe Grooß ${ }^{1}$, Ines Tritscher $^{1}$, Michael Höpfner ${ }^{3}$, \\ Michael Pitts ${ }^{4}$, Andrew Orr $^{5}$, and Martin Riese ${ }^{1}$ \\ ${ }^{1}$ Forschungszentrum Jülich, Institut für Energie und Klimaforschung, Stratosphäre, IEK-7, Jülich, Germany \\ ${ }^{2}$ Forschungszentrum Jülich, Jülich Supercomputing Centre, Jülich, Germany \\ ${ }^{3}$ Karlsruhe Institut für Technologie, Institut für Meteorologie und Klimaforschung, Karlsruhe, Germany \\ ${ }^{4}$ NASA Langley Research Center, Hampton, VA, USA \\ ${ }^{5}$ British Antarctic Survey, Cambridge, UK
}

Correspondence: Reinhold Spang (r.spang@fz-juelich.de)

Received: 28 September 2017 - Discussion started: 27 October 2017

Revised: 9 March 2018 - Accepted: 13 March 2018 - Published: 16 April 2018

\begin{abstract}
The Michelson Interferometer for Passive Atmospheric Sounding (MIPAS) instrument aboard the European Space Agency (ESA) Envisat satellite operated from July 2002 to April 2012. The infrared limb emission measurements provide a unique dataset of day and night observations of polar stratospheric clouds (PSCs) up to both poles. A recent classification method for PSC types in infrared (IR) limb spectra using spectral measurements in different atmospheric window regions has been applied to the complete mission period of MIPAS. The method uses a simple probabilistic classifier based on Bayes' theorem with a strong independence assumption on a combination of a well-established two-colour ratio method and multiple 2-D probability density functions of brightness temperature differences. The Bayesian classifier distinguishes between solid particles of ice, nitric acid trihydrate (NAT), and liquid droplets of supercooled ternary solution (STS), as well as mixed types.

A climatology of MIPAS PSC occurrence and specific PSC classes has been compiled. Comparisons with results from the classification scheme of the spaceborne lidar CloudAerosol Lidar with Orthogonal Polarization (CALIOP) on the Cloud-Aerosol-Lidar and Infrared Pathfinder Satellite Observations (CALIPSO) satellite show excellent correspondence in the spatial and temporal evolution for the area of PSC coverage $\left(A_{\mathrm{PSC}}\right)$ even for each PSC class. Probability density functions of the PSC temperature, retrieved for each class with respect to equilibrium temperature of ice and based on coincident temperatures from meteorological re-
\end{abstract}

analyses, are in accordance with the microphysical knowledge of the formation processes with respect to temperature for all three PSC types.

This paper represents unprecedented pole-covering dayand nighttime climatology of the PSC distributions and their composition of different particle types. The dataset allows analyses on the temporal and spatial development of the PSC formation process over multiple winters. At first view, a more general comparison of $A_{\mathrm{PSC}}$ and $A_{\mathrm{ICE}}$ retrieved from the observations and from the existence temperature for NAT and ice particles based on the European Centre for MediumRange Weather Forecasts (ECMWF) reanalysis temperature data shows the high potential of the climatology for the validation and improvement of PSC schemes in chemical transport and chemistry-climate models.

\section{Introduction}

The essential role of polar stratospheric clouds (PSCs) in the depletion of stratospheric ozone has been well established (e.g. Solomon, 1999). Heterogeneous reactions on PSC particles convert chlorine reservoir species (in particular $\mathrm{HCl}$ and $\mathrm{ClONO}_{2}$ ) to chlorine radicals that destroy ozone catalytically. Although PSCs have been explored now for more than 30 years, there are still many open questions that limit our ability to accurately simulate the formation and surface area of different PSC types and consequently limit the quality of 
the prediction of future polar ozone loss rates in a changing climate system (e.g. Peter and Grooß, 2012). For example, although the closure of the Antarctic ozone hole is projected to occur by the end of 21st century, large uncertainties exist in the rate and timing of its recovery (e.g. Eyring et al., 2013; WMO, 2014; Fernandez et al., 2017).

PSCs are located in the cold polar vortices in both winter hemispheres and consist of three types of particles, which can also coexist: supercooled ternary solution (STS) droplets, nitric acid hydrates (most likely nitric acid trihydrate, NAT), and ice particles (e.g. Lowe and MacKenzie, 2008). Their formation and existence are mainly determined by the temperature with an ice frost point $\left(T_{\mathrm{ICE}}\right)$ at $\sim 188 \mathrm{~K}$ and an equilibrium NAT temperature $\left(T_{\mathrm{NAT}}\right)$ of $\sim 196 \mathrm{~K}$ (at $55 \mathrm{hPa}$, for $5 \mathrm{ppmv} \mathrm{H}_{2} \mathrm{O}$ and $10 \mathrm{ppbv} \mathrm{HNO}_{3}$ ). Due to nucleation barriers, temperatures lower than the existence temperatures are required. A possible nucleation pathway for NAT needs pre-existing ice particles and therefore temperatures $3-4 \mathrm{~K}$ below $T_{\text {ICE. }}$ STS droplets grow with decreasing temperatures from binary to ternary solution droplets. Temperatures, where the $\mathrm{HNO}_{3}$ weight percentages are as large as $\mathrm{H}_{2} \mathrm{SO}_{4}$ and the volume increases strongly, are described with $T_{\text {STS }}$ $(\sim 192 \mathrm{~K})$. The formation of STS droplets is well understood (e.g. Carslaw et al., 1995), but for NAT and ice particles, new formation mechanisms by heterogeneous nucleation on meteoric smoke are under discussion (e.g. Hoyle et al., 2013; Engel et al., 2013; Grooß et al., 2014). The formation of NAT requires much lower temperatures, usually $3-4 \mathrm{~K}$ below the ice frost point, on pre-existing ice particles or at higher temperatures on meteoritic dust particles (e.g. Peter and Grooß, 2012; Hoyle et al., 2013).

PSCs impact polar chemistry in three ways: (1) through heterogeneous reactions, where the rates depend on surface area, particle, and typology of the particle (Sander et al., 2011; Shi et al., 2001); (2) through the uptake of $\mathrm{HNO}_{3}$ into PSCs thereby reducing $\mathrm{HNO}_{3}$ concentrations in the gas phase temporarily (e.g. Salawitch et al., 1989); and (3) through NAT particles growing to large-enough sizes for sedimentation, so that a permanent removal of the gas-phase $\mathrm{HNO}_{3}$ from polar air masses (denitrification) can occur (e.g. Fahey et al., 2001; Molleker et al., 2014) and has a significant impact on the Antarctic and Arctic polar ozone loss (e.g. Waibel et al., 1999). In addition, extensive dehydration by sedimentation of PSC ice particles is a regular appearance in the Antarctic stratosphere.

Difficulties in making accurate predictions on the ozone recovery in a changing climate stems from a variety of problems in chemistry-climate models (CCMs). One important problem is the poor representation of PSCs. CCMs used for assessments of polar stratospheric ozone loss (e.g. Eyring et al., 2013) often employ rather simple PSC schemes. Although these simple schemes today mostly include reactions on all types of PSCs, the simplifications may lead to a NATdominated heterogeneous chemistry. However, it is known that heterogeneous chemistry on supercooled ternary solu- tion and on cold binary aerosol particles probably dominates polar chlorine activations (e.g. Solomon, 1999; Drdla and Müller, 2012; Kirner et al., 2015). The models usually do not include comprehensive microphysical modules to describe the evolution of different types of PSCs over the winter. Additional, mesoscale temperature variations caused by orographic gravity waves can be crucial for the formation of PSCs, especially for conditions close to the temperature threshold for particle formation (Carslaw et al., 1998; Dörnbrack et al., 2002; Engel et al., 2013; Hoffman et al., 2017), but are missing from the current generation of CCMs (Orr et al., 2015).

Assumptions on the occurrence of different types of PSCs in CCM simulations have been shown to only have a limited impact on many aspects of polar ozone loss in case studies for both the Arctic and Antarctic. For example, liquid PSC particles alone are sufficient to simulate nearly all of the ozone loss using current model chemistry (Wohltmann et al., 2013; Kirner et al., 2015; Solomon et al., 2015). However, which type of PSCs is present at the top of the ozone loss region (between 10 and $30 \mathrm{hPa}$ ) is important, as shown for Antarctica by Kirner et al. (2015). Further, in the Arctic polar vortex of the 2009/2010 winter, the initial activation occurred in PSCs covering only a small portion of the vortex; under such conditions, the type of PSC present can be decisive (Wegner et al., 2016).

Heterogeneous reaction rates on PSCs and cold binary aerosols are always strongly temperature dependent, but the reaction rate for a particular heterogeneous reaction also depends on the PSC particle type (e.g. Drdla and Müller, 2012; Wegner et al., 2012). Further, although reaction rates have been adjusted in recent model studies (e.g. Wegner et al., 2012), there is still a substantial uncertainty on the rates of heterogeneous reactions on NAT (Carslaw et al., 1997; Wegner et al., 2012) which makes determining the type of PSC present in the atmosphere important.

Consequently, measurements of the particle type of PSCs are highly desirable but are so far limited. Ideally, these measurements should cover the complete polar vortex and should last for multiple winters, which is only possible either passively in the mid-infrared or actively through lidar measurements from space. Climatologies of PSC observations and their classifications in PSC types over several winters are so far restricted to ground-based stations - usually lidar systems (e.g. Di Liberto et al., 2014; Achtert and Tesche, 2014) or are based on spaceborne solar occultation measurements (e.g. Hervig et al., 1997; Fromm et al., 2003) usually without or with only very limited PSC type information (Strawa et al., 2002). Consequently, these datasets are restricted to local sites or are directly attached to the solar terminator and therefore do not allow measurements inside the cold and dark winter polar vortices. UV-vis limb scattering measurements can also measure PSC distributions (von Savigny et al., 2005) but currently do not allow PSC types to be discriminated and are restricted to daylight conditions. Stellar occultation mea- 
surements in the UV-vis wavelength region can partly compensate for the difficulty of adequate coverage of the polar night cap (e.g. Vanhellemont et al., 2010) but are so far not able to differentiate PSC types.

Limb infrared emission measurements have the advantage of being available during day- and nighttime which allows unrestricted sounding of both polar vortices (e.g. Spang et al., 2001). With a certain spectral resolution of the measurement, it is even possible to discriminate between specific PSC types (Spang and Remedios, 2003; Spang et al., 2004). The restricted time series of polar winter measurements by the Improved Stratospheric and Mesospheric Sounder (ISAMS) (Taylor et al., 1994), the Cryogenic Limb Array Etalon Spectrometer (CLAES) (Massie et al., 1994), and the Cryogenic Spectrometers and Telescopes for the Atmosphere (CRISTA) (Spang et al., 2001) already showed the high potential of infrared (IR) limb measurements in the field of PSC research. A more complete picture of PSC morphology and composition on polar vortex-wide scales is emerging from a suite of recent satellite missions. The Michelson Interferometer for Passive Atmospheric Sounding (MIPAS) (Fischer et al., 2008) on Envisat (2002-2012) and the Cloud-Aerosol Lidar with Orthogonal Polarization (CALIOP) (Winker et al., 2009) on the Cloud-Aerosol-Lidar and Infrared Pathfinder Satellite Observations (CALIPSO) satellite (2006-present) retrieve PSC information from space beyond comparison with respect to horizontal, vertical, and temporal resolution and coverage. These datasets have already motivated numerous PSC studies that both extend and challenge our present knowledge of PSC processes and modelling capabilities (e.g. Peter and Grooß, 2012, and references therein).

CALIOP analyses of multiple winters of PSC particle type distributions in a climatological sense are presented by Pitts et al. $(2009,2011,2013)$. These studies stressed the scientific potential comprehensive multi-annual PSC climatologies would have. PSC analyses of MIPAS were so far restricted to individual winters (Spang et al., 2005a, b) or specific case studies (Höpfner et al., 2006b; Eckermann et al., 2009). A better latitudinal coverage than CALIOP up to the pole as well as homogenous day- and nighttime coverage by MIPAS provide substantial additional information on climatological PSC distributions. Furthermore, MIPAS data are available for the time period from July 2002 to May 2006, before CALIOP became operational. Consequently, one of the main objectives of the actual Stratosphere-troposphere Processes And their Role in Climate (SPARC) PSC activity is to synthesise and archive the CALIOP and MIPAS long-term datasets in combination with former in situ balloon borne (Antarctic and Arctic) and remote lidar (Antarctic) measurements into a state-of-the-art PSC climatology. Here, we present one part of the planned combined PSC climatology, comprising a new MIPAS PSC dataset covering the full mission lifetime of 10 years from July 2002 to March 2012.

The paper is structured as follows. Section 2 introduces MIPAS and other involved instruments. Section 3 provides a summary of the MIPAS detection and classification method more extensively presented in Spang et al. (2016) - given in conjunction with a comparison to CALIPSO and plausibility tests for the results of the classification approach. Section 4 describes in detail various aspects of the climatology, like temporal and spatial evolution of the PSC type distribution. In addition, first analyses of retrieved parameters from the climatology which are important for the evaluation of chemistry transport models (CTMs) and chemistry-climate models (CCMs) are presented, for example, time series of the area of the hemisphere covered by PSCs $\left(A_{\mathrm{PSC}}\right)$ and the three corresponding PSC types ( $A_{\mathrm{ICE}}, A_{\mathrm{NAT}}$, and $\left.A_{\mathrm{STS}}\right)$.

\section{Instruments and datasets}

\subsection{MIPAS instrument on Envisat}

The MIPAS instrument aboard the Envisat satellite measured limb infrared spectra in the wavelength range from 4 to $15 \mu \mathrm{m}$ (Fischer et al., 2008) from July 2002 to April 2012. The satellite operated in a Sun-synchronous orbit (inclination $98.4^{\circ}$ ) and allowed geographical coverage up to both poles due to additional poleward tilt of the primary mirror (usually up to $87^{\circ} \mathrm{S}$ and $89^{\circ} \mathrm{N}$ ). Due to technical problems with the interferometer in 2004, the exceptionally high spectral resolution of $0.025 \mathrm{~cm}^{-1}$ (HR: high-resolution mode) was reduced to $0.0625 \mathrm{~cm}^{-1}$ (OR: optimised-resolution mode) (Raspollini et al., 2013). The processed level $1 \mathrm{~b}$ radiance data from the measurement period of July 2002 to March 2004 (phase 1) were measured in the HR mode and from January 2005 to March 2012 (phase 2) in the OR mode. Also the vertical and horizontal sampling for the nominal measurement modes were changed from phase 1 to 2 . A fixed vertical step size of $3 \mathrm{~km}$ up to a tangent height of $\sim 42 \mathrm{~km}$ was used in phase 1 and changed to an altitude-dependent vertical step size of $1.5-4.5 \mathrm{~km}$ for phase 2 , with increasing altitude steps with height. In addition, the lowest tangent altitude was changed from a constant value to latitude-dependent values of $5 \mathrm{~km}$ at the poles up to $12 \mathrm{~km}$ over the Equator in phase 2. The horizontal sampling was improved from 550 to $420 \mathrm{~km}$.

The trapezoidal form of the vertical field of view (FOV) of MIPAS has a base width of $4 \mathrm{~km}$ and a top width of $\sim 2.8 \mathrm{~km}$. In a cross-track direction, the FOV covers a range of $30 \mathrm{~km}$. In the following analyses, version 5 level $1 \mathrm{~b}$ data, processed and provided by the European Space Agency (ESA), have been used as the underlying dataset for cloud detection and classification of the PSCs by composition. More details on detection and classification are given in Sect. 3.

\subsection{CALIOP instrument on CALIPSO}

The CALIOP instrument is a dual wavelength polarisationsensitive lidar that provides high vertical resolution profiles of backscatter coefficients at 532 and $1064 \mathrm{~nm}$ (Winker et al., 2009). CALIOP is the primary instrument on the 
CALIPSO satellite, which flies in a $98^{\circ}$ inclination Sunsynchronous orbit at an altitude of $705 \mathrm{~km}$. This orbit geometry facilitates nadir-viewing measurements up to latitudes of $82^{\circ} \mathrm{N} / \mathrm{S}$. CALIOP PSC analyses are based on nighttimeonly CALIPSO Level 1B $532 \mathrm{~nm}$ parallel and perpendicular backscatter coefficient measurements smoothed to a uniform $5 \mathrm{~km}$ horizontal (along track) by $180 \mathrm{~m}$ vertical grid over the altitude range from 8.2 to $30 \mathrm{~km}$.

This study uses data from the CALIPSO Lidar Level 2 Polar Stratospheric Cloud Mask version 2.0 (Pitts et al., 2018), in which PSCs are detected as statistical outliers relative to the background aerosol population in either $532 \mathrm{~nm}$ perpendicular backscatter coefficient or $532 \mathrm{~nm}$ scattering ratio (the ratio of total to molecular backscatter). To facilitate detection of optically thin clouds, successive horizontal averaging $(5,15,45$, and $135 \mathrm{~km})$ is applied to the data to improve signal-to-noise ratio (SNR) (Pitts et al., 2009). Based on comparison of measurements with theoretical optical calculations for non-equilibrium liquid-NAT or liquidice mixtures, CALIOP PSCs are classified by composition into five categories: STS, (liquid-) NAT mixtures, enhanced NAT mixtures (those liquid-NAT mixtures with very high NAT number densities), ice, and wave ice (ice PSCs induced by mountain waves). Version 2.0 CALIOP PSC data are significantly improved over the original version, especially in the separation of NAT mixtures and ice in the presence of denitrification and dehydration.

\section{PSC detection and classification}

The PSC detection and classification approach used for MIPAS is described in detail in Spang et al. (2012), which presents the MIPclouds processor for detection and cloud parameter retrievals, and in Spang et al. (2016), which also introduces the methodology of the Bayesian classifier for PSC types and represents the results of the classification in comparison to the CALIOP classification. In the following, we only summarise the major principles and characteristics of the applied methodology.

\subsection{MIPAS detection of PSCs}

The MIPclouds processor and its data products are used for the detection of cloudy spectra in each profile of the measurement period. A step-like data processing approach of up to five detection methods was chosen for the processor to provide summary cloud top height (CTH) information with the best possible detection sensitivity (Spang et al., 2012). The most common detection method for IR limb measurements is the cloud index (CI) colour ratio approach with constant detection threshold (Spang et al., 2001, 2004), but also variable CI threshold profiles changing with altitude, latitude, and month were combined (Spang et al., 2012; Sembhi et al., 2012).
The $\mathrm{CI}$ is a ratio of the mean radiances at a wavelength of $796 \mathrm{~cm}^{-1}$, dominated by $\mathrm{CO}_{2}$ emissions, and around $832 \mathrm{~cm}^{-1}$, an atmospheric window region. The CI has been extensively used for the detection of cloud-contaminated spectra for various types of IR limb measurements of spaceand airborne instruments (e.g. Spang et al., 2001, 2004, 2007) and has been established as a simple, robust, and, depending on the applied threshold value, highly sensitive cloud detection method. The CI is well suited for the detection of PSCs, because it correlates extremely well with the integrated particle volume or area density along the limb path (VDP/ADP), and also with optical depth and extinction. ADP or VDP are the integrated particle size distribution quantities where the IR limb signal of a cloud is most sensitive (Spang et al., 2012). The long path length through the atmosphere enables to detect extremely low volume and area densities if the cloud has a certain horizontal extent (several kilometres). For example, a 1 or $100 \mathrm{~km}$ horizontally extended ice cloud is detectable for ice water contents of $>0.3$ and $>0.003 \mathrm{mg} \mathrm{m}^{-3}$, respectively (Spang et al., 2015).

In addition, a retrieval approach with simplified assumptions for the radiative transfer in clouds (Hurley et al., 2011) was implemented in the MIPclouds detection algorithm. This retrieval copes with the difficulty of using a $3 \mathrm{~km}$ vertical FOV to determine a more realistic CTH inside the FOV. We use the step-like approach of all detection methods and the summary CTH for selecting the first cloud-affected spectrum in an altitude scan for starting with the PSC classification for this and all potentially cloudy tangent heights below.

\subsection{Bayesian classifier for IR limb measurements}

Figure 1 shows a schematic overview of the Bayesian classifier approach applied in this study. Basis for the classification is an extensive database of more than 600000 modelled PSC spectra (Spang et al., 2012) with varying PSC types: ice, NAT, and STS ( $n_{\text {type }}=3$ ), with variable microphysical (volume density, median radius of the particle size distribution) and macrophysical (horizontal and vertical extent) parameters for the modelled cloud scenes. Mixed-type clouds have not been considered in the radiative transfer calculation. Mean radiances of seven wavelength regions, mainly atmospheric window regions, where cloud emissions can contribute significantly to the measured signals, were selected for the computation of two colour ratios, CI and so-called NAT index (NI), as well as five brightness temperature differences (BTDs).

The NI is a colour ratio of the 820 to $796 \mathrm{~cm}^{-1}$ wavelength region, with the latter region already used in the CI approach. It is a measure for enhanced peak-like emission at $\sim 820 \mathrm{~cm}^{-1}$ by a characteristic spectral feature in the NAT emission spectrum (Höpfner et al., 2002; Spang and Remedios, 2003). These emissions are strongly enhanced and are clearly detectable in NAT cloud spectra for median radii $r<3 \mu \mathrm{m}$ (Höpfner et al., 2006a). The combination of CI 
and NI, like illustrated in the probability density function (PDF) (I) in Fig. 1, was the first sophisticated method to attribute IR limb spectra to specific PSC types. The three highlighted regions (1-3) separate ice in region (1) from NAT with $r<3 \mu \mathrm{m}$ in region (2) and most likely STS but also large NAT particle or optically very thin ice clouds in region (3). The CI-NI approach was first applied by Spang and Remedios (2003) to the CRISTA southern polar winter observations in August 1997. Although the method has some weaknesses in the differentiation of ice and STS as well as difficulties to discriminate between cloud emissions in the IR spectra dominated by STS particles with emissions by large NAT particles $(r>3 \mu \mathrm{m})$, the method has been used and improved in various studies and with different instruments (Spang et al., 2005a, b; Höpfner et al., 2006a, b; Arnone et al., 2012; Lambert et al., 2012).

Measurements of large NAT particles, so-called NAT rocks, are of specific interest because it is the particle type causing denitrification in the polar vortex (Fahey et al., 2001; Molleker et al., 2014). Measurements - in situ as well as remote sensing - of these particle are very difficult and quite rare in the literature. In addition, the formation process of NAT and NAT rocks in the polar stratosphere is still under debate (e.g. Peter and Grooß, 2012), and consequently more sophisticated information on PSC particle type and size are desirable.

To improve the classification and to bypass specific weaknesses of the CI-NI approach, in total four classification diagrams $(i=1 \ldots m$, with $m=4)$ have been selected by Spang et al. (2016). These combine different sensitivities with cloud composition and optical thickness (depth) of the clouds and are retrieved from the cloud scenario database of the MIPclouds study (Spang et al., 2012) and the MIPAS measurements themselves (e.g. the applicable range in the parameter space of the correlation diagrams is guided by the PDF of the measurements). All classification diagrams show distinctive regions (in total 13) which can be attributed to a specific composition of PSCs or potentially mixtures of PSC types. The four methods are then combined in a simple probabilistic classifier based on applying Bayes' theorem with strong (naive) independence assumptions. Based on the radiative transfer calculations covering the full parameter space of PSCs in the database and the MIPAS measurements themselves, a priori-like probabilities are defined for each classification region. Examples of monthly probability density functions of the MIPAS measurements for each classification diagram are presented in Fig. 1 for July 2010. For a single spectrum, the normalised product probability $\left(P_{j}\right.$, with $j=1 \ldots n$ and $n=3$ ) for each PSC type is computed, and stepwise decision criteria are defined:

- If the maximum of $P_{j}$ (with $j=1 \ldots 3$ ) $>50 \%$, then type $(j)$ is the most likely PSC composition.
- If two $P_{j}$ values are between 40 and $50 \%$, then a mixedtype class, for example, ICE_STS for $j=1$ and $j=3$, is attributed.

- If the classification results in $P_{1}, P_{2}$, or $P_{3}<40 \%$, then the cloud spectrum is unclassified and pigeon-holed to the class "unknown".

Analyses with the version 1.2.8 Bayesian Classifier for MIPAS PSC observation showed that the mixed-type classes ICE_STS and ICE_NAT have an insignificant partitioning $(<0.1 \%$ per month) with respect to all cloud classes and are negligible for further statistical analyses (Spang et al., 2016). On the other hand, the STS_NAT class can show larger occurrence rates (5-10\% per month) especially for the Southern Hemisphere (SH) early winter conditions (see Sect. 3.5).

Spang et al. (2016) demonstrated the overall reliability of the results of the new classification method by detailed sensitivity tests on the Bayesian classifier input parameter and extensive comparisons with the CALIOP lidar classification. The latter comparison worked on a large statistical basis with narrow coincidence criteria and showed an overall good agreement between the partitioning of the defined PSC classes but also some significant mismatches. Mismatches highlight differences between the two instruments (MIPAS and CALIOP) with respect to the detection sensitivity for specific PSC types (and mixtures of them) due the very different measurement technique and geometry (Spang et al., 2016).

It should be noted that MIPAS and CALIOP have completely different sensitivities to measure cloud structures with a mixture of PSC types. It is quite plausible that PSCs will usually include mixtures of all three particle types (ice, NAT, and STS) (Pitts et al., 2013) dependent on the temperature and available trace gas concentrations $\left(\mathrm{HNO}_{3}\right.$ and $\left.\mathrm{H}_{2} \mathrm{O}\right)$, but the intensity of the analysed measurement signals, for example, NI for MIPAS or the backscatter ratio for CALIOP, can have quite different sensitivities to detect one specific type in a mixture of PSC types in the measurement volume. Limb measurements are characterised by a horizontally extended measurement volume (hundreds of kilometres). The line of sight will usually pass various regions dominated by different particle types. Consequently, the effects of mixtures are very difficult to quantify and are only partly considered in the PSC classification of MIPAS and CALIOP.

\subsection{Retrieved parameter and data processing}

The main macrophysical cloud retrieval parameter is the cloud top height information and a cloud mask of flags, where for each cloudy spectrum at and below the CTH and down to tangent heights of $12 \mathrm{~km}$ the classification result is presented. For the version 1.2.8 Bayesian classifier, the following cloud mask of PSC classes is defined: -1 : unclassified (non-cloudy), 0: unknown, 1: ice, 2: NAT, 3: STS, 4: ICE_NAT, 5: STS_NAT, and 6: ICE_STS. The classification 


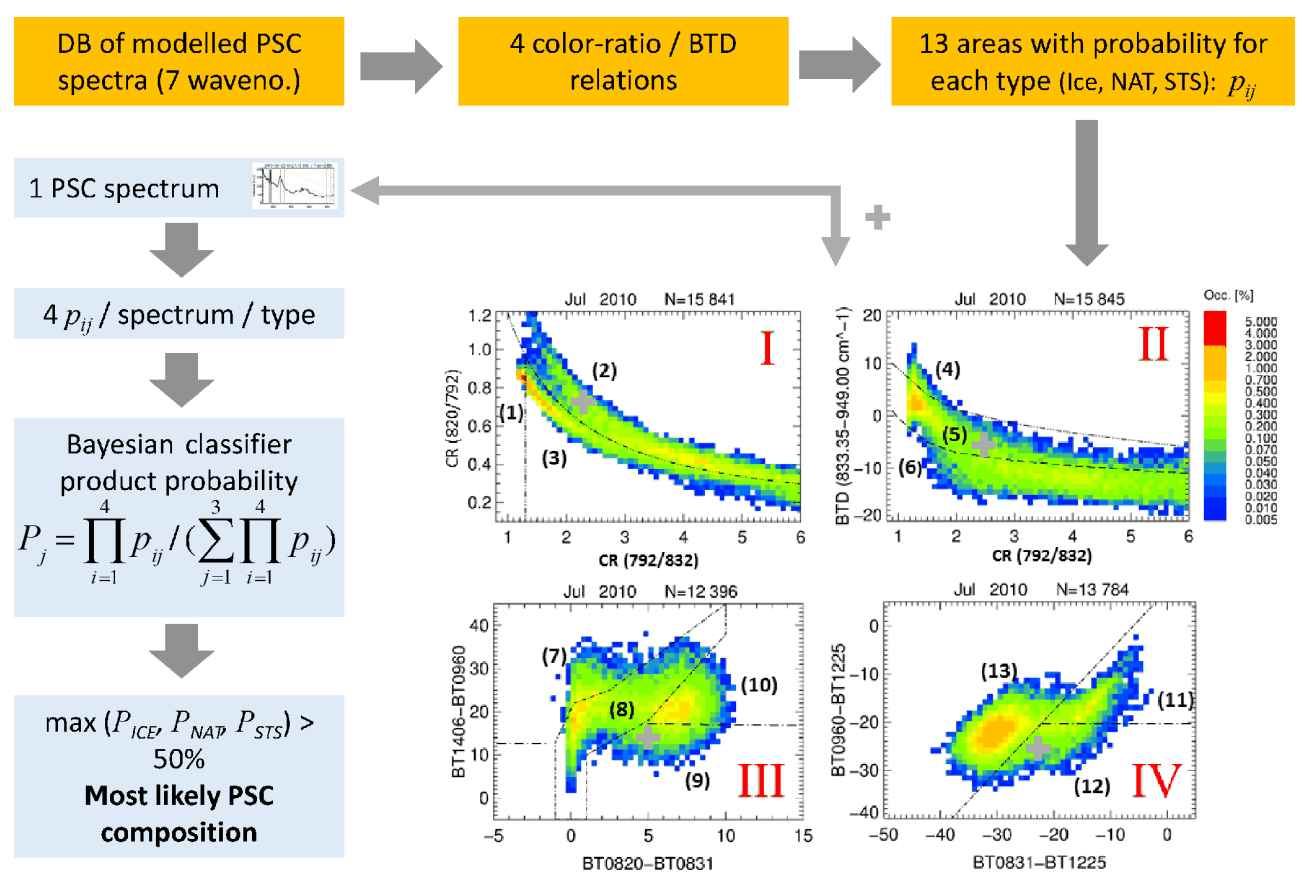

Figure 1. Schematic diagram of the Bayesian classifier procedure (blue boxes) for the MIPAS PSC type classification. Orange boxes illustrate the part of the huge database (DB) of modelled PSC spectra for the computation of colour ratios and BTDs. The diagrams I to IV are MIPAS examples of monthly probability density functions for SH July 2010 data applied in the Bayesian classifier. The grey cross in each of the four diagrams is illustrating the input information of a single PSC spectrum, which is used to retrieve the most likely PSC composition (for details, see text and Spang et al., 2016).

is only applied to spectra with $\mathrm{CI}<6$ at or below the first tangent height level of detection. This more stringent $\mathrm{CI}$ threshold compared to the CI-based detection methods using the step-like detection algorithm of MIPclouds gives the opportunity to classify spectra which are most likely cloudy but are only detected by the most sensitive methods and may be missed in the combined MIPclouds detection approach mainly optically very thin cloud layers (Spang et al., 2012). This may introduce some biases in the statistics by falsepositive events. However, this risk occurs only at the lower bound of the cloud layer (cloud base height) and for detections close to the signal-to-noise level (altitudes $>30 \mathrm{~km}$ ). These can easily be excluded by a restriction in altitude from the following analyses.

There is a general caveat to separate emissions in the IR by large NAT particles from emissions by STS particles due to their similar behaviour (Spang et al., 2016). Consequently, we cannot exclude larger abundance of large NAT particles $(r>3 \mu \mathrm{m})$ in cloud events classified with STS by the Bayesian classifier, and the term STSmix is probably a better description for this type of MIPAS PSC class. Unfortunately, there is no proper approach to quantify or validate this uncertainty. For example, the CALIOP classification has also weaknesses in differentiation of large NAT particles from small NAT and STS particles adequately, which does not allow us to use CALIOP as a transfer standard for MIPAS.
Additional meteorological information on the coincident temperature, pressure, and potential temperature based on ERA-Interim data (ERAi) (Dee et al., 2011) is also merged into the dataset for simplicity of statistical analyses with the PSC dataset. Additional information on the geographical location, time, and satellite position per spectrum is part of the data structure as well as parameters retrieved from MIPAS measurements and used in the detection and classification algorithm (e.g. CI and NI for each measured spectrum), and are saved in the Network Common Data Form (NetCDF) output files of the data processing scheme. Details on the set of parameters stored in the output files (PSC related and auxiliary parameter) are presented in Sect. 6.

The complete Envisat mission with MIPAS data from July 2002 to April 2012 has been processed with the Bayesian classifier. There are data gaps between July and September 2007, and extended data gaps up to months of no data between April and December 2004 followed by a period with reduced temporal coverage between January and December 2005. During this period, the repeat cycle of measurements per week was increased in a stepwise process from 50 back to $100 \%$. From 2006 onwards, a very homogeneous spatial and temporal coverage has been achieved until the loss of contact to Envisat on 8 April 2012. The resulting 10 winter seasons in the Northern Hemisphere (2002/2003 to 2011/2012) and nine seasons in the Southern Hemisphere (2002, 2003, and 2005 to 2011) represent a unique dataset of 
day- and nighttime PSC type measurements up to the poles for process studies in the polar winter stratosphere. This is especially important for the evaluation and improvements of CCMs and CTMs for a better predictability of long-term ozone trends in a changing climate.

\subsection{Examples of the Bayesian classifier results}

The usefulness of the new PSC classification algorithm has already been presented in Spang et al. (2016) and Hoffmann et al. (2017). These studies showed that some of the MIPAS PSC detections are directly linked to small-scale temperature fluctuations due to gravity waves (GWs). Figure 2 shows two examples for the horizontal distribution of the PSC classes of the Bayesian classifier for a single day in January 2010 for the Northern Hemisphere (NH) and June 2011 in the $\mathrm{SH}$ in an altitude region defined by the potential temperature range $\Theta=500 \mathrm{~K} \pm 20 \mathrm{~K}$. Both examples highlight very special events and characteristics of the classifier dataset. The NH example shows an exceptionally large synoptic area of ice clouds north of Scandinavia surrounded by the usually more frequent observations of NAT and STS clouds in this region, as temperatures are usually significantly higher than $T_{\mathrm{ICE}}$. Note that the Montgomery stream function contour lines suggest that downstream of this region NAT particles exist, which have been "seeded" in the ice cloud area and may have formed on pre-existing ice.

The example for the $\mathrm{SH}$ in June 2010 shows a typical PSC type distribution for this time of the winter, which involves the widespread occurrence of NAT particles usually around the middle of June. The map shows indications for the development of the so-called NAT belt (Tabazadeh et al., 2001). Höpfner et al. (2006b) and Eckermann et al. (2009) showed that this kind of distribution is mainly composed of small NAT particles $(r<3 \mu \mathrm{m}$, which are triggered by strong mountain wave (MW) activity over the Antarctic Peninsula. The Antarctic Peninsula is a well-known hot spot region for mountain waves (e.g. Wu and Jiang, 2002; Hoffmann et al., 2017), and the link between specific mountain waves events and the NAT formation process has been analysed by Hoffmann et al. (2017) in more detail with MIPAS dataset and Atmospheric Infrared Sounder (AIRS) GW observations. The study of Hoffmann et al. (2017) found many events (> 50), where the PSC occurrence was clearly linked to MW activity. There are additional MW source regions in Antarctica where this link is also observed, e.g. the Transantarctic Mountains, but the Antarctic Peninsula is by far the most prominent region. MWs may play a prominent role in the total abundance of PSCs, especially in the NH where the temperatures are more often close to but not significantly below the threshold temperatures for PSC formation. Consequently, the link between MWs and GW sources in general needs special emphasis in CCMs and CTMs for a better accuracy in the prediction of the future polar ozone in a changing climate (Orr et al., 2015).

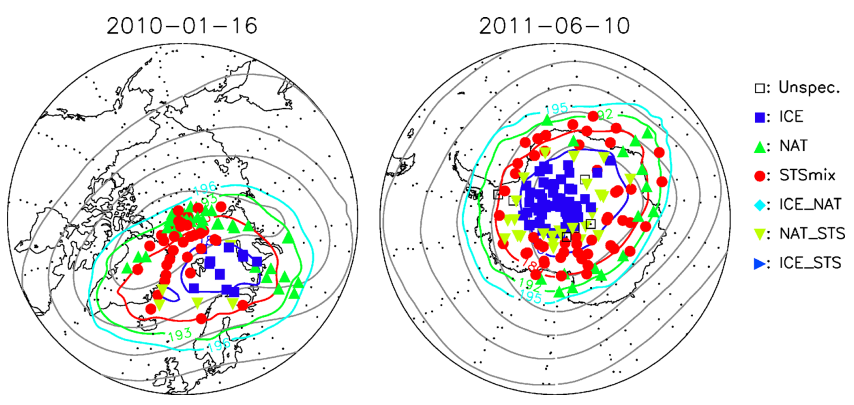

Figure 2. Examples of the horizontal distributions of MIPAS PSC composition classes for $\mathrm{NH}$ winter and $\mathrm{SH}$ winter conditions at an altitude level of $500 \mathrm{~K}$ potential temperature $( \pm 20 \mathrm{~K})$. In addition to the different cloud-type symbols described in the figure legend (see main text for details), temperature contours for $T_{\mathrm{ICE}}$ (dark blue) (Marti and Mauersberger, 1993), $T_{\mathrm{STS}}=\left(T_{\mathrm{NAT}}+T_{\mathrm{ICE}}\right) / 2$ (red), $T_{\text {NAT }}$ (green) (Hanson and Mauersberger, 1988), and $T_{\text {NAT }}+2 \mathrm{~K}$ (turquoise) based on ERA-Interim data (Dee et al., 2011) with constant water vapour and nitric acid mixing ratios, grey contours of the Montgomery stream function at $500 \mathrm{~K}$, and black dots for noncloudy MIPAS profile locations are superimposed.

\subsection{Temperature probability distribution of PSC types}

Temperature frequency distributions of various classified PSC classes are a useful tool for testing qualitatively the reliability of classification methods (e.g. Pitts et al., 2013) and are extremely valuable for the analysis of PSC formation processes and their chemical imprint by heterogeneous reaction on the polar ozone layer (Pitts et al., 2013; Lambert et al., 2012). Mean SH and NH winter histograms are presented in Figs. 3 and 4 related to $\Delta T=T-T_{\mathrm{ICE}}$ for all measured PSC spectra between 16 and $30 \mathrm{~km}$ and selected six SH and four NH polar winters, respectively. As well as showing considerable interannual variability for both regions, the frequency distributions for the southern polar winter differ quite considerably from those for the northern polar winter.

The ice type histograms for the SH show the coldest and narrowest distribution, with its maxima slightly below or just at $T_{\text {ICE }}$. Arctic winter conditions are usually not cold enough to produce comparable maxima to STS or NAT like in the Antarctic, but a few winters (e.g. 2009/2010 and 2010/2011) show a certain activity in ice formation.

The STS class is usually dominating the NH distribution, due to generally warmer vortex conditions. The temperature of the maximum of the frequency distribution for STS is $\sim 3-$ $4 \mathrm{~K}$ above $T_{\mathrm{ICE}}$, which is in line with the equilibrium temperatures of STS and ice (e.g. Carslaw et al., 1995). However, for specific meteorological conditions, like in winters 2009/2010 and 2010/2011, the NAT population has a similar proportion to the "warm" SH winters, e.g. for the winter of 2010 (De Laat and Van Weele, 2011; see also Sect. 4.1.2).

STS and NAT both show very similar shape in the frequency distribution for the $\mathrm{SH}$ and $\mathrm{NH}$ (maximum location, $\Delta T_{\max }$, and width) but a slightly shifted maximum for NAT 
to $T-T_{\text {ICE }}>5 \mathrm{~K}$ in the $\mathrm{NH}$. The latter maximum might be affected by the rare occasions of temperatures below $T_{\mathrm{ICE}}$ in the NH. Therefore, heterogeneous NAT nucleation on preexisting ice particles can only dominate the NAT formation in the $\mathrm{SH}$, whereas in the NH heterogeneous NAT formation at $T>T_{\text {ICE }}$ may play a dominant role. Recent analyses of in situ and satellite measurements together with new formation mechanisms in microphysical models support this so far excluded formation pathway at $T>T_{\text {ICE }}$ (Hoyle et al., 2013). Despite the ongoing debate over homogeneous and heterogeneous nucleation, an analogous analysis by Pitts et al. (2013) for CALIOP data and the independent classification scheme shows nearly identical relations between the STS and the mixed classes of CALIOP (mixtures of STS with contributions of NAT particles depending on the number densities and particle sizes of NAT).

The mixed-type class NAT_STS shows very asymmetric frequency distribution with a shift to the cold tail of STS $(\mathrm{SH} / \mathrm{NH})$ and NAT $(\mathrm{SH})$. These "cold tail" classes might indicate the coexistence of STS and NAT particles formed by heterogeneous nucleation just around $T_{\text {ICE }}$. However, IR measurements of small NAT particles usually show the characteristic NAT spectral feature at $820 \mathrm{~cm}^{-1}$ (Höpfner et al., 2002; Spang and Remedios, 2003). Distributions for these events, selected with the former CI-NI classification approach (see Sect. 3.2, Spang and Remedios, 2003; Höpfner et al., 2006a), are highlighted in Figs. 3 and 4 by the black line. The CI-NI distributions show no obviously enhanced number for NAT events at corresponding $\Delta T_{\max }$ of the NAT_STS class but a significant enhancement in the centre region for the NAT class (most likely an overestimation). Nevertheless, we cannot exclude from this comparison that NAT_STS distribution might be part of the CI-NI distribution. In addition, the CI-NI class shows a significantly larger number of events than the Bayesian classifier-based NAT events $(\sim 20 \%)$. Frequently, very weak signatures of the NAT feature may be classified as ice or STS due to characteristic radiance contribution of these types in other wavelength regions. These spectra can appear in regions of the two-parameter scatter distributions II to IV of Fig. 1 with higher probabilities for STS or ice than for NAT.

Overall, the behaviour in the $T-T_{\text {ICE }}$ histograms is in line with the current understanding of the formation of PSCs in the winter polar vortices, which gives us confidence that the Bayesian classifier is a reliable approach providing data for more detailed scientific studies on the formation processes of PSCs as well for a climatology of PSC composition of the entire MIPAS measurement period.

A similar temperature analysis of the PSC classes by Pitts et al. (2013, Figs. 8 and 9) with the CALIOP lidar data shows comparable results with respect to the maxima location of the frequency distribution for ice ( $T-T_{\mathrm{ICE}} \sim-1$ to $\left.0 \mathrm{~K}\right)$ and a systematic shift to warmer temperatures for the other PSC classes. However, the shift, for example, for the CALIOP STS events, is only in the range of $1-2 \mathrm{~K}$, but the MIPAS analysis shows a shift of $\sim 4 \mathrm{~K}$. In addition, the CALIOP $T-T_{\text {ICE }}$ histograms show significantly smaller widths in the distribution than the MIPAS analysis. The typical difficulty for limb sounders to define the exact position of the cloud along the line of sight $(\sim 400 \mathrm{~km})$ will raise additional uncertainties in the cloud temperature assignment, where the coincidence of the tangent point with the ERAi temperature information is applied. The CALIOP PSC observations have the advantage over MIPAS of coincident $\mathrm{H}_{2} \mathrm{O}$ and $\mathrm{HNO}_{3}$ measurements from the Microwave Limb Sounder (MLS) on the Earth Observing System (EOS) Aura satellite with virtual zero miss time and miss distance (e.g. Lambert et al., 2012). To compensate for this difficulty, we applied MLSbased zonal mean water vapour measurements in equivalent latitude coordinates to the MIPAS tangent point equivalent latitude. Yet, both approximations for the MIPAS analysis will produce significant noise-like scatter in the histograms and consequently a broader distribution width than in the CALIOP analyses.

\subsection{Comparison of retrieved $A_{\mathrm{PSC}}$ with CALIOP/CALIPSO}

CALIOP classes of PSC types in version 1.0 (https://eosweb.larc.nasa.gov/project/calipso/lidar_12_ polar_stratospheric_cloud_table, last access: 13 October 2017) have been compared with the Bayesian classifier results (version 1.2.8) for MIPAS by Spang et al. (2016). Here, we present a first comparison of the daily and height-resolved area of the hemisphere covered by PSC ( $A_{\mathrm{PSC}}$, in $10^{6} \mathrm{~km}^{2}$ units), a secondary data product based on the classification results for both instruments where for CALIOP the newer version (2.0) has been used (see Sect. 2.2). Due to the sparse along-track sampling of MIPAS $(400-500 \mathrm{~km})$ and the need for an adequate sampling statistics for the computation of $A_{\text {PSC }}$, we defined eight equally area-spaced latitude bands from $55^{\circ}$ up to the pole, with $2.3^{\circ}(250 \mathrm{~km})$ up to $12.2^{\circ}(1340 \mathrm{~km})$ width in latitude. In addition, a vertical grid with $1 \mathrm{~km}$ step size and $2 \mathrm{~km}$ grid-box size for MIPAS and $180 \mathrm{~m} / 180 \mathrm{~m}$, respectively, for CALIOP, has been defined. For each vertical grid cell and latitude band, the mean occurrence frequency of each PSC class has been calculated. Finally, the mean over the eight latitude bands multiplied by the total area of the polar cap $\left(\operatorname{area}>55^{\circ} \mathrm{N} / \mathrm{S}=46 \times 10^{6} \mathrm{~km}^{2}\right)$ is representing a quantitative measure of the real PSC coverage. This approach bypasses the caveat of the irregular sampling density due to the orbit geometry and avoids artefacts like the overestimated ice partition with respect to the other classes of the Bayesian classifier reported in Spang et al. (2016).

Rex et al. (2004) showed a surprisingly good correlation of the volume of PSCs $\left(V_{\mathrm{PSC}}(T)\right)$ with the degree of Arctic ozone loss. Based on meteorological reanalyses (mainly temperature), $V_{\mathrm{PSC}}(T)$ is defined as the stratospheric volume where conditions are cold enough $\left(T<T_{\mathrm{NAT}}\right)$ for the exis- 

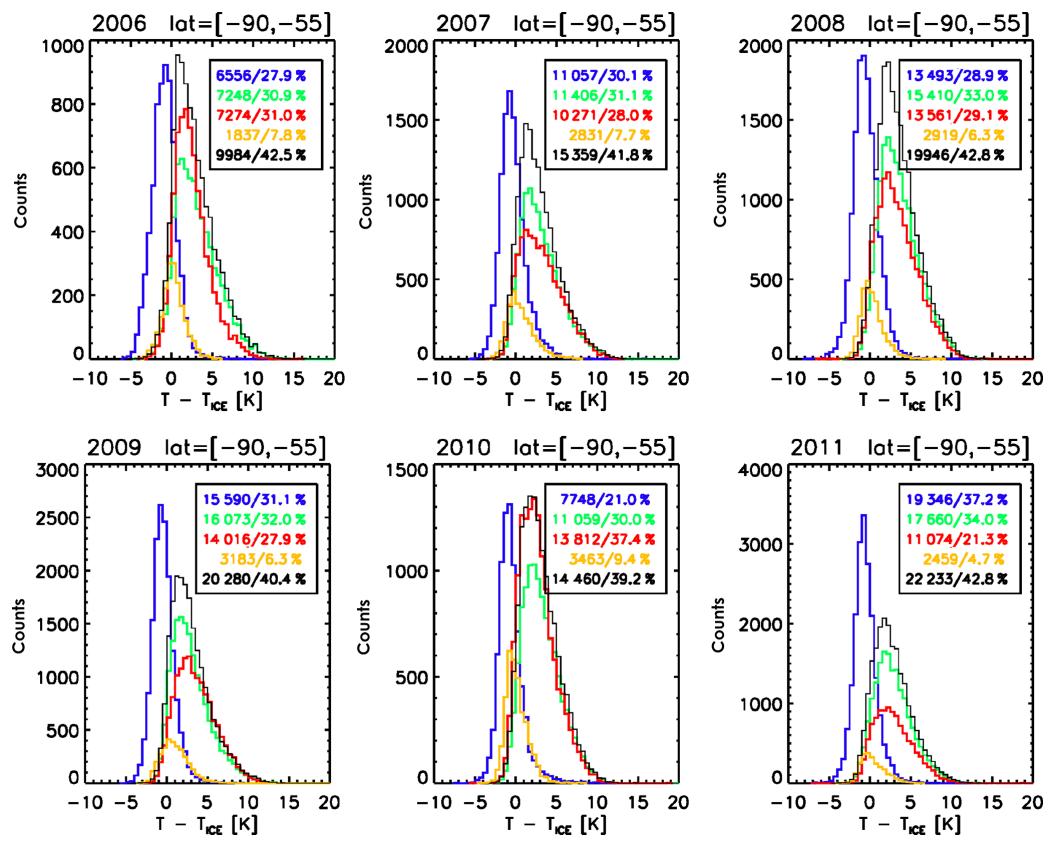

Figure 3. The 6 years of southern polar winter PSC class frequency distributions as a function of $T-T_{\text {ICE }}$. For consideration of the dehydration process in the polar vortex over the winter, coincident temperatures from ERA-Interim reanalyses and zonal mean water vapour mixing ratios on equivalent latitudes of the Microwave Limb Sounder (MLS) instrument (e.g. Lambert et al., 2012) are used for the computation of $T_{\text {ICE }}$ (Marti and Mauersberger, 1993). The years 2006-2011 are presented for the four main PSC classes ice (blue), NAT (green), STS (red), and NAT_STS (orange), as well as a former NAT classification method (black) based on Spang and Remedios (2003); for details, see the text. Please note the changing maximum counts of observation and corresponding maximum value of the $y$ axis for individual winters. The coloured numbers in the legend indicate the total number of the corresponding cloud types and the partition on the total number of PSC observations in percent.

tence of PSCs and is computed by simple integration of corresponding $A_{\mathrm{PSC}}(T)$ over certain altitude layers of the meteorological analyses. Because this quantity is not the real volume or area of PSC coverage, the term should be better described with potential volume of PSC formation. $V_{\mathrm{PSC}}(T)$ has been used as a proxy for ozone destruction $\left(\Delta \mathrm{O}_{3}\right)$ potential and for the validation of CCMs and CTMs over multiple winters and the prediction capabilities of CCMs in a changing climate. However, using $V_{\mathrm{PSC}}$ as a proxy for polar ozone depletion has limitations, because even for the same definition for $V_{\mathrm{PSC}}$ (which is to some extent arbitrary) the resulting proxy depends on the employed analysis or reanalysis scheme (e.g. Rex et al., 2004; Rieder and Polvani, 2013). This demonstrates that patterns of $V_{\mathrm{PSC}}$ derived from PSC observation - put forward here - constitute an important quantity for evaluating the performance of CCMs. Both satellite datasets allow for the first time comparisons of these $\Delta \mathrm{O}_{3}$ proxies based on meteorological analyses with the true atmospheric state of measured $A_{\mathrm{PSC}}$ and $V_{\mathrm{PSC}}$ (see also Sect. 4.2).

For a first impression on the validity of both datasets, we compare in Fig. 5 the CALIOP and MIPAS APSC evolution for the SH winter 2009. The MIPAS measurements need to be restricted to a maximum latitude of $82^{\circ} \mathrm{S}\left(82^{\circ} \mathrm{N}\right.$ for $\mathrm{NH}$ winter), the maximum latitude where CALIOP can take mea- surements. A quantitative comparison is only possible by applying this restriction; otherwise, the very high probability to measure ice PSCs in the SH vortex core for mid-winter conditions would bias the statistics due to the lack of CALIOP measurements at these high latitudes (see also Spang et al., $2016)$. For the individual cloud classes $\left(A_{\text {TYPEi }}\right)$, STS for MIPAS compared to STS for CALIOP, MIPAS NAT to the sum of NAT mixtures and enhanced mixtures, and MIPAS ice to the CALIOP ice plus wave ice class, we found generally good consistency in the temporal evolution. The NAT class shows in both measurements the largest coverage of the polar cap. The onset of the PSC 2009 season is characterised by first outbreaks of STS detection in mid-May for both datasets, followed by first NAT occurrences between the end of May and beginning of June. All classes show the characteristic descending of the PSC area throughout the course of the winter. The declining ice probability in September with a slight recovery in mid-September is nicely shown by both instruments.

Even the absolute values of $A_{\text {TYPEi }}$ for specific days and altitudes show a remarkably good agreement over the entire winter, although some differences are also apparent. For example, the vertical extent for PSC type occurrence shows systematic offsets on the lower bound due to two effects: (a) the 

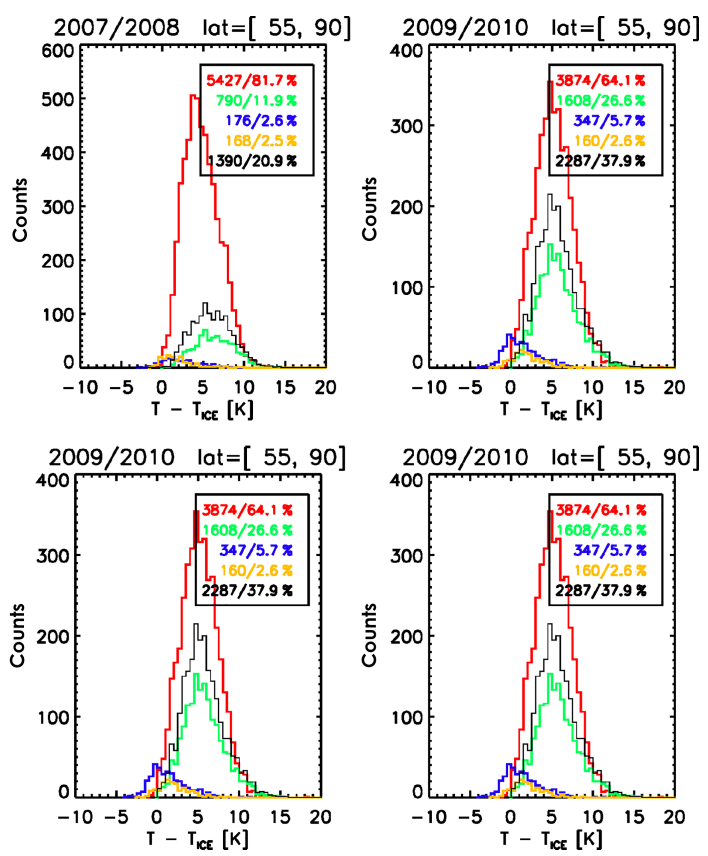

Figure 4. The 4 years of $\mathrm{NH}$ polar winter PSC type frequency distributions with respect to $T_{\text {ICE }}(2007 / 2008,2009 / 2010,2010 / 2011$, and 2011/2012) using the analogue presentation of Fig. 3. Please note the changing scale of observation counts for each winter.

MIPAS analysis is truncated below the $12 \mathrm{~km}$ altitude level using a $1 \mathrm{~km}$ step size for vertical grid, and (b) if the analysis is not based on a full radiative transfer retrieval of the measured radiance profile (e.g. an extinction retrieval), then the limb technique has limitations in quantifying accurately the cloud bottom information (Spang et al., 2012). For the presented analysis, we used all spectra flagged as cloudy up to $6 \mathrm{~km}$ below the actual CTH. In comparison to CALIOP, where the cloud base height is usually well defined in the measurements, the MIPAS restriction shows a tendency to underestimate the PSC bottom altitude and consequently the lower edge in vertical coverage for each PSC type in Fig. 5. Note that the CALIOP and MIPAS detection algorithms are also sensitive to cirrus clouds in the tropopause region. In May, CALIOP and especially MIPAS show clouds classified as NAT at $12 \mathrm{~km}$, which might be an artefact of the algorithms. It should be mentioned that CALIOP detects far more ice than NAT clouds in this altitude region, and that the total coverage for NAT plus ice of both instruments is in good agreement. This indicates that cirrus clouds at the tropopause are the most likely explanation for these detections. For so far unknown reasons, these early winter cloud events at the tropopause are in most cases misclassified as NAT for MIPAS. In addition, the MIPAS measurements show cloud detections at higher altitudes than CALIOP (up to $16 \mathrm{~km}$ ) for this early winter period. The large FOV of MIPAS likely causes these unexpected and potentially overestimated cloud tops at and above the polar tropopause. Optical thick cirrus clouds in the lowest part of the FOV create overestimation in cloud top height of up to 1.5 to $2 \mathrm{~km}$ for IR limb sounders (Spang et al., 2012, 2015). The vertical grid box size of 2 $\mathrm{km}$ for MIPAS compared to $180 \mathrm{~m}$ for CALIOP causes also a slight overestimation in the cloud top occurrence statistics for MIPAS.

\section{The MIPAS PSC climatology}

In the following sections, we present details of the new MIPAS PSC climatology. The climatology is the first polecovering, day- and nighttime dataset that differentiates particle composition. Together with the upcoming CALIOPbased PSC composition climatology and pole-covering measurements of relevant trace gases for stratospheric ozone chemistry from the MLS instrument (e.g. Lambert et al., 2012) and from MIPAS (e.g. Arnone et al., 2012), a unique database for polar process studies and climatological aspects of the PSC distribution with respect to ozone destruction is now available under variable winter conditions for both hemispheres.

\subsection{Overall PSC occurrence}

\subsubsection{Hemispheric coverage of mean PSC occurrence}

The climatological mean of all PSC occurrences for nine SH and $10 \mathrm{NH}$ winter seasons is presented in Fig. 6. We limited the analysis to the altitude region at $16-24 \mathrm{~km}$ where PSCs tend to occur, and computed the occurrence frequency by dividing the number of cloudy spectra by the total number of spectra for an equal space of $10^{\circ}$ longitude $\times 5^{\circ}$ latitude grid boxes.

Both hemispheres show very different distributions. Compared to the $\mathrm{NH}$, the PSC coverage is significantly larger for the $\mathrm{SH}$ and the maximum occurrence frequencies are more than a factor of 4 higher ( $\sim 40 \%$ versus $10 \%)$. In addition, the maximum PSC location is centred over the pole for the SH but is remarkably shifted over the Barents Sea and northern Scandinavia for the $\mathrm{NH}$, which is in line with a shift of the climatological mean temperature contours and minimum in the same region. This shift is caused by stronger planetary wave activity for $\mathrm{NH}$ winters with strong wave number 1 amplitudes. Consistently, the climatological mean temperature in the $\mathrm{SH}$ show 10 to $15 \mathrm{~K}$ lower mean temperatures than the NH polar vortex. These results are in line with the state-of-the-art physical and chemical understanding of polar processes.

To illustrate the temporal development with respect to the geographical location over the entire winter season, Fig. 7 shows the climatological mean of PSC occurrence on a monthly basis. The PSC season in the SH comprises MaySeptember and is longer and more intensive than the equivalent December-February season in the NH (March is not presented as the PSC occurrence is minimal). The SH shows 

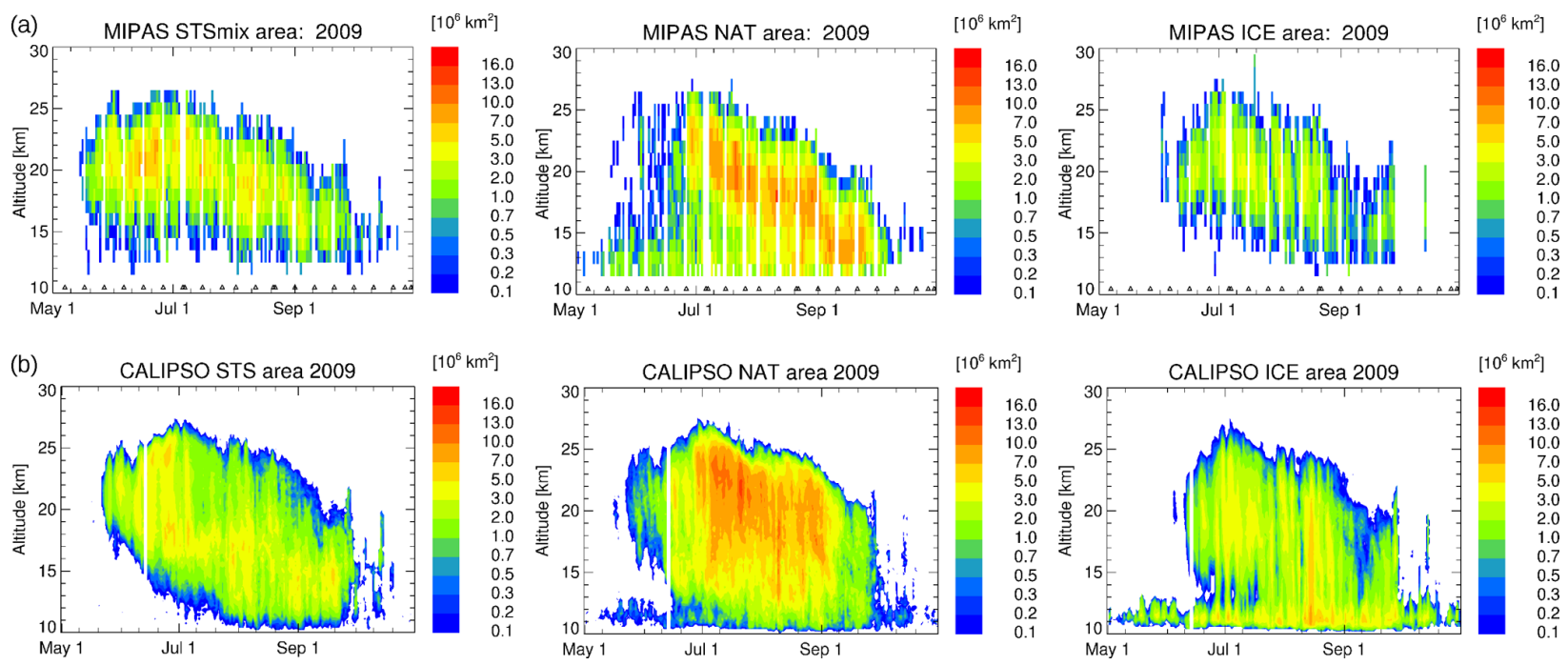

Figure 5. PSC type classification comparison between the MIPAS (Bayesian classifier version 1.2.8) row (a) and CALIOP (V2) row (b) based on the identical computation of $A_{\mathrm{PSC}}$ for each dataset. For better comparability, MIPAS observations are restricted to latitudes $<82^{\circ} \mathrm{S}$, the latitude coverage of CALIOP. Black triangles at $10.5 \mathrm{~km}$ altitude in the MIPAS time series highlight data gaps usually caused by the mesosphere/thermosphere measurement mode.
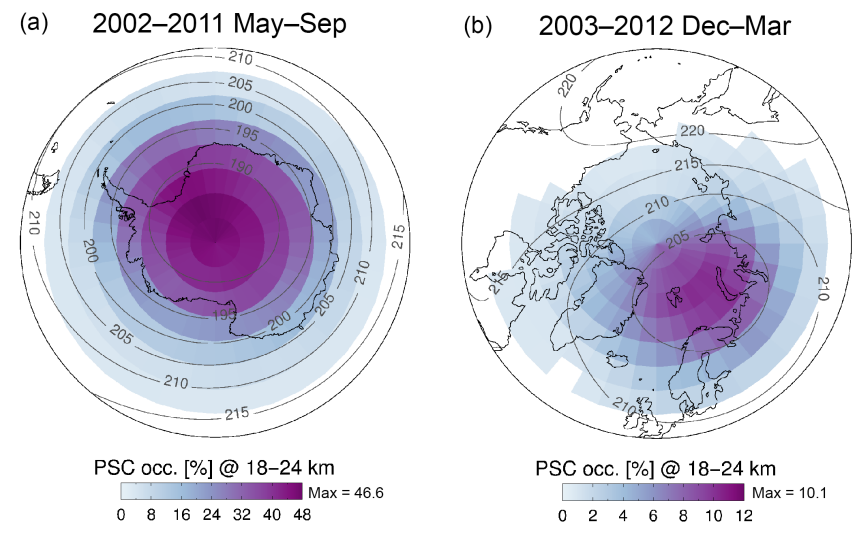

Figure 6. Occurrence of PSC spectra with respect to the total number of measured spectra in the complete MIPAS measurement period for $\mathrm{SH}$ (a) and $\mathrm{NH}$ (b) polar winter conditions. A mean for May-September and December-March for 2002-2011 of ERAibased temperature contours at $50 \mathrm{hPa}$ is superimposed in grey. Please note the different ranges of the colour bar for both statistics.

the typically concentric distribution for all months with the PSC occurrence rates maximum at or close to the pole and in close correspondence with the temperature contours $\left(T_{\min }\right)$. In the $\mathrm{NH}, \mathrm{PSC}$ occurrence rates are more variable from year to year, usually with at most several weeks of severe PSC activity distributed in the December-March period. A change of the maximum location from the January to the February mean from the Greenland Sea to the east in the direction of the north of Russia is mainly caused by individual years with colder stratospheric winters with unusually large occurrence frequencies and where the geographical location of the plan- etary wave 1 minimum can be quite different from year to year or can even change over the winter.

\subsubsection{Interannual variability in PSC coverage}

Similar to the altitude-resolved $A_{\text {PSC }}$ presentation in Fig. 5, it is common for a better characterisation of a specific winter to compare the temporal evolution of $V_{\mathrm{PSC}}$ or $A_{\mathrm{PSC}}$ at a single altitude level with a climatological mean of the parameter and its variability (e.g. Tilmes et al., 2004). These simple parameters allow also to estimate the ozone destruction potential of a single winter (Rex et al., 2004). However, these time series are based on the assimilated temperature distribution from meteorological reanalyses, representing the thresholds for PSC existence. However, the actual PSC and PSC-type coverage might be quite different.

Here, instead of $V_{\mathrm{PSC}}$, we present for MIPAS the maximum area covered by PSC spectra in the altitude range 15 to $30 \mathrm{~km}$. This integrated view to the PSC distribution allows us to bypass the difficulties of passive IR limb sounders to retrieve accurate cloud bottom information, which is necessary for the derivation of $V_{\mathrm{PSC}}$ by measurements. Accurate cloud bottom information is especially a problem for optically thicker cloud conditions (e.g. Spang et al., 2012). Nevertheless, this quantity is a good estimate of the actual area and overall PSC coverage of the polar vortex, and can also easily be computed from CTM and CCM model output fields for comparisons and evaluation purposes.

Figure 8 shows the temporal evolution of the integrated maximum $A_{\text {PSC }}\left(A_{\text {PSCmax }}\right)$ for each winter season (May to October) in the MIPAS measurement period (2002 to 2012) for the SH and NH. The winter of 2006 showed, with max- 


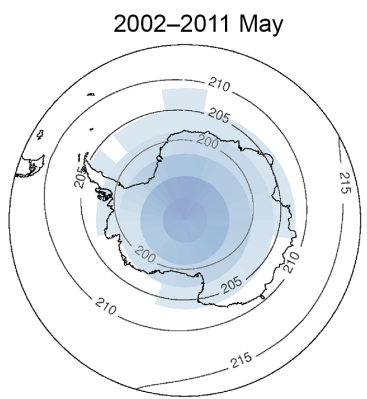

PSC occ. [\%] @ 18-24 km

$0 \quad 102030 \quad 405060$

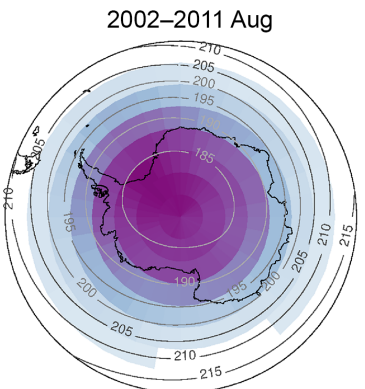

PSC occ. [\%] @ 18-24 km

$0 \quad 102030405060$

2003-2012 Dec

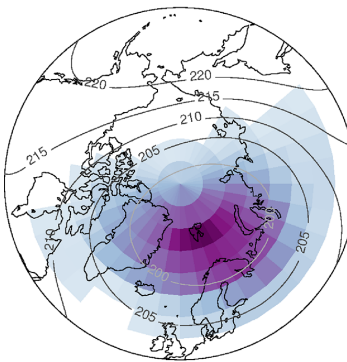

PSC occ. [\%] @ 18-24 km \begin{tabular}{lllllll}
\hline & 3 & 6 & 9 & 12 & 15 & 18
\end{tabular}

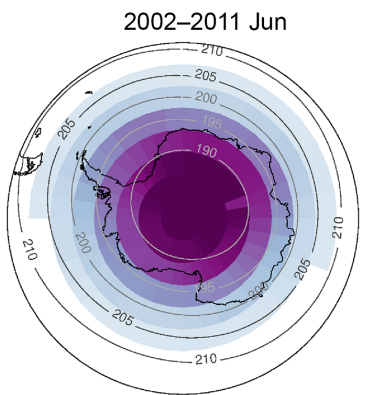

PSC occ. [\%] @ 18-24 km $0 \quad 102030405060$

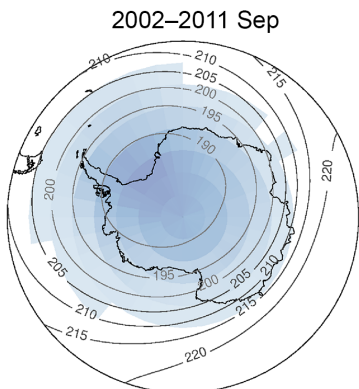

PSC occ. [\%] @ 18-24 km $0 \quad 102030 \quad 405060$

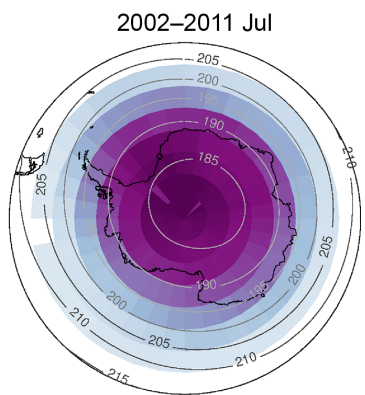

PSC occ. [\%] @ 18-24 km 0102030405060

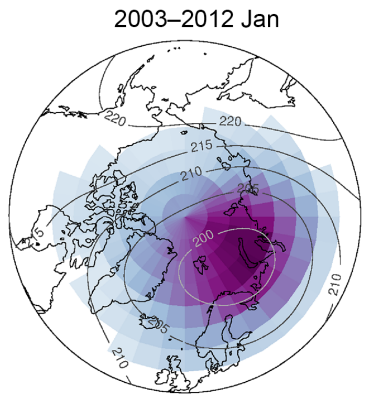

PSC occ. [\%] @ 18-24 km

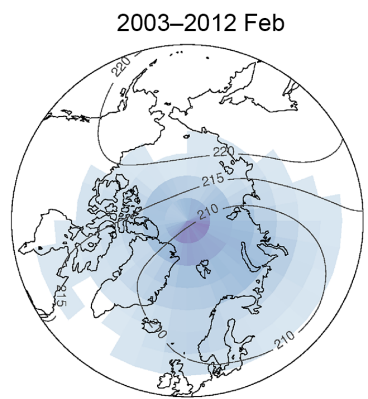

PSC occ. [\%] @ 18-24 km

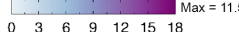

Figure 7. Monthly mean of hemispheric PSC occurrence between 18 and $24 \mathrm{~km}$ for the months with PSC activity (SH: May, June, July, August, and September; NH: December, January, and February). A mean of 2002-2012 ERAi-based temperature contours at 50 hPa is superimposed in grey. Please note the different ranges in the colour code between SH and NH observations.

imum values of up to 25 million $\mathrm{km}^{2}$ corresponding to more than $60 \%$ of the polar cap south of $55^{\circ} \mathrm{S}$, the largest cloud area extent. The interannual variability is small for the $\mathrm{SH}$ compared to the $\mathrm{NH}$ winters. The absolute values of $A_{\mathrm{PSC} \text { ax }}$ in the $\mathrm{NH}$ are significantly smaller than in the $\mathrm{SH}$ (factors of 2-3) and periods of PSC occurrences are restricted to several weeks compared to long extended periods of a minimum of 4 months (2002) and maximum close to 6 months (2006 and 2011) for the SH.

The winter of 2002 showed at the end of September an exceptional major warming event in the $\mathrm{SH}$ stratosphere, where the polar vortex split into two vortices and reunited again (e.g. Allen et al., 2003). Temperatures in the strato- sphere were higher than usual during most of this winter, caused by strong planetary wave activity in the troposphere already in May, and several minor warmings took place during August and September. These very unusual conditions are well reproduced in the PSC activity measured by MIPAS. Although the measurements started late and are partly interrupted due to instrumental tests during the commissioning phase of the satellite, $A_{\mathrm{PSCmax}}$ for 2002 shows the lowest values of all nine SH winter seasons. A significant cut in early August (minor warming event, partly masked by a data gap) is followed by only a slight recovery in PSC activity, with still exceptionally low values compared to other winters; a second breakdown takes place in early Septem- 

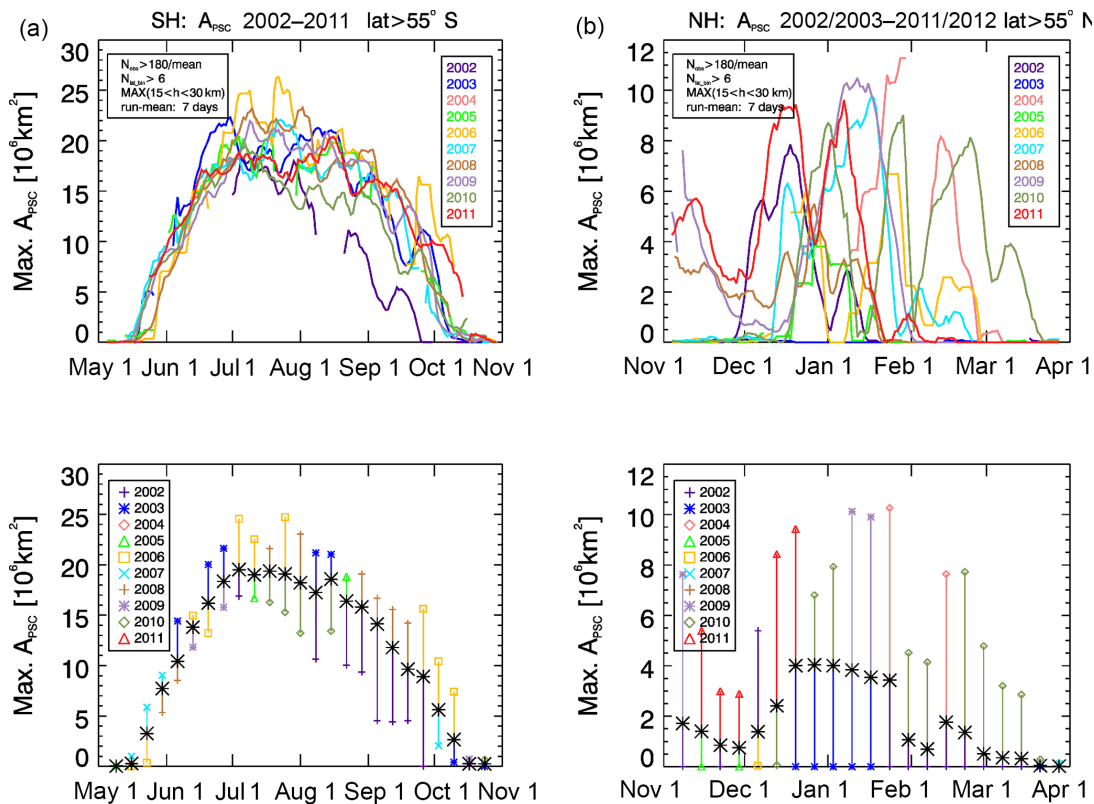

Figure 8. Maximum MIPAS area of PSC for SH (May-October, a) and NH winter conditions (November-March, b) computed as the running mean over 7 days through all seasons (top row). In addition, weekly maximum/minimum (colour-coded symbols) and mean values (* symbol) of $A_{\text {PSC }}$ are presented in the bottom row. For both types of representations, each season is colour coded, where for the NH the year in the figure legend (for example, 2002) is linked to the potential PSC season November 2002-March 2003, respectively. For the NH results, minimum values of zero appear for several years and the symbols are overlaid.

ber, and finally the PSCs completely disappear in the second half of September (usually in early October), coincident with the major warming event starting in mid-September, ending with a final warming on 25-26 September 2002 (Butler et al., 2017). In addition to the 2002 winter, the PSC 2010 season shows remarkably low $A_{\text {PSCmax }}$ values between the end of July and mid-October. De Laat and van Weele (2011) found anomalously warm temperatures in MLS data for this specific Antarctic winter and discuss in detail the role of minor stratospheric warmings in 2010.

For the NH observations, the winter of 2010/2011 was exceptional in the sense of three unusual periods of PSC activity from late December to mid-March. The winter of 2011/2012 is highlighted by a very early and extended PSC coverage. The 2003/2004 season showed even no PSC activity at all. Special attention has to be taken to the unexpected local maxima in $A_{\text {PSCmax }}$ in November for the years 2008/2009, 2009/2010, and 2011/2012. All these periods are strongly influenced by an enhanced aerosol load of the upper troposphere/lower stratosphere (UTLS) region caused by volcanic eruptions. Dedicated MIPAS aerosol analyses (Griessbach et al., 2016) show that the PSC seasons 2008/2009, 2010/2011, and 2011/2012 are influenced by the volcanic eruptions of Kasatochi (Günther et al., 2018), Sarychev (Wu et al., 2017), Grimsvötn, and Nabro (Griessbach et al., 2016; Günther et al., 2018) in August 2008, June 2009, May 2011, and July 2011, respectively. Due to the fact that the Bayesian classifier so far does not include a differentiation between background or volcanic aerosols and PSCs, these events have been treated and classified as PSC particles. The artificial enhanced PSC activity is observed from the lowest altitude level around the tropopause $(12 \mathrm{~km})$ up to maximum heights of $\sim 16 \mathrm{~km}$ depending on the strength and penetration depth of the corresponding volcanic eruption (see also Sect. 4.2.4).

\subsection{Temporal and spatial analysis of the PSC classes}

In addition to the overall PSC occurrence over the winter, the results of the Bayesian classifier allow a detailed analysis of the horizontal and vertical distribution of individual types of PSCs in the climatological mean or for specific winters.

\subsubsection{Monthly means}

Figure 9 represents an example for winter 2009 (MaySeptember) of the monthly mean occurrence frequencies between 18 and $24 \mathrm{~km}$ altitude for the individual classes (STS, NAT, ice, and NAT_STS in each rows) of the classifier. A $10^{\circ}$ longitude $\times 5^{\circ}$ latitude grid is used for the statistics.

The different temporal onset of each PSC type becomes obvious here again (see also Fig. 5). STS dominates the early winter followed by NAT and ice in June/July. The ice is concentrated on the centre of the cold polar vortex surrounded by a belt-like structure for NAT. This is similar to the plots of individual days presented in Figs. 2 or 11 in Hoffmann 

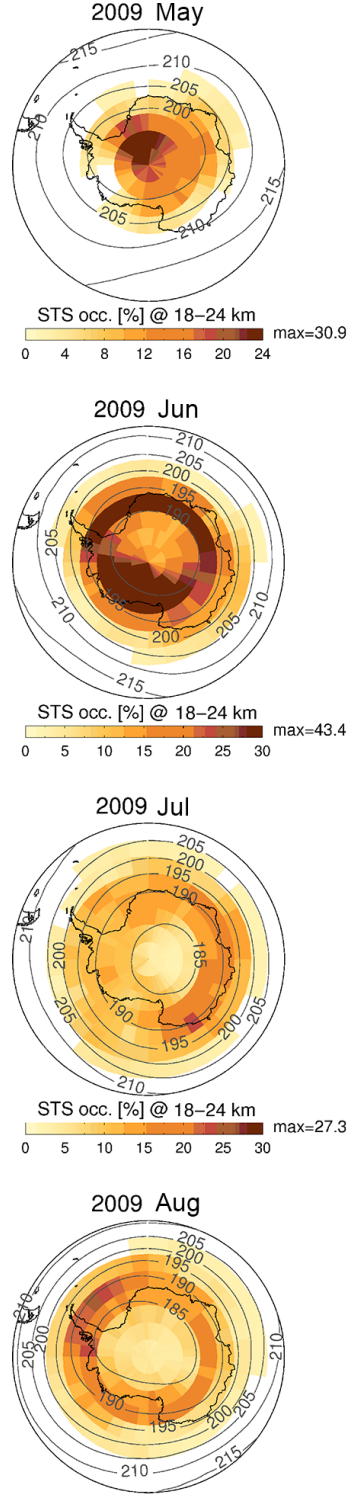

STS occ. [\%] @ 18-24 km $\max =25.2$

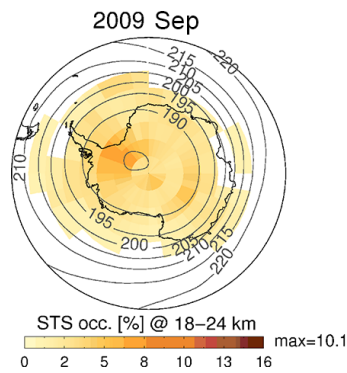

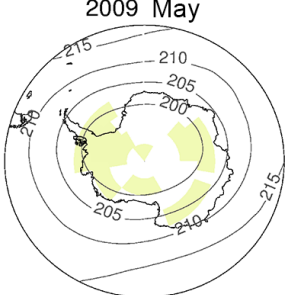
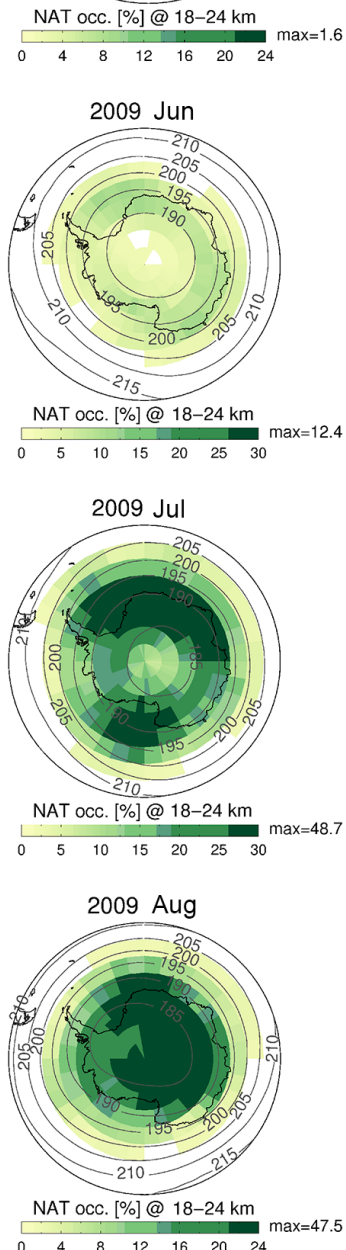

$\max =47.5$

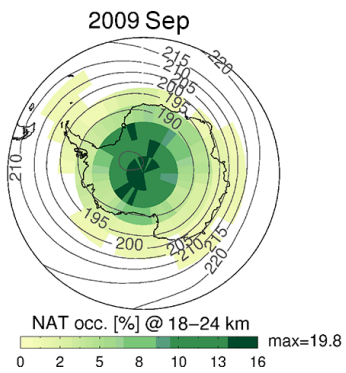

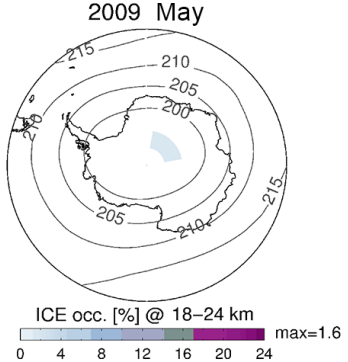

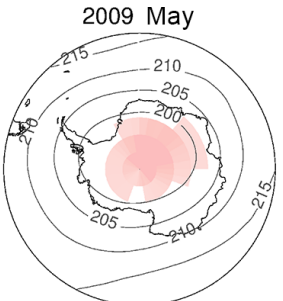

NAT_STS occ. [\%] @ 18-24 km
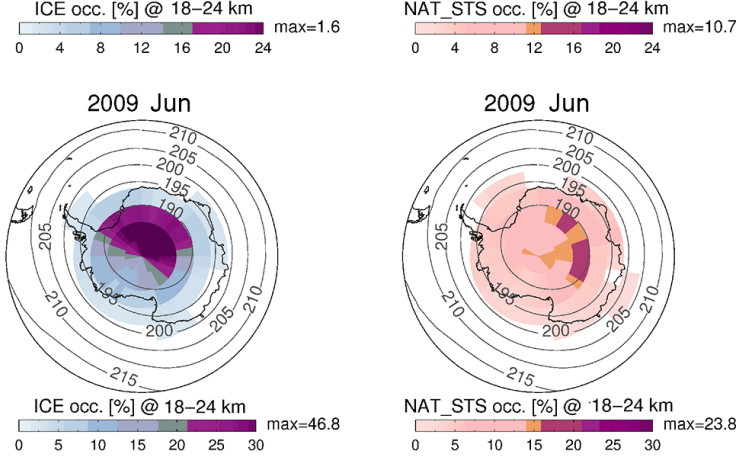

NAT_STS occ. [\%] @ 18-24 km
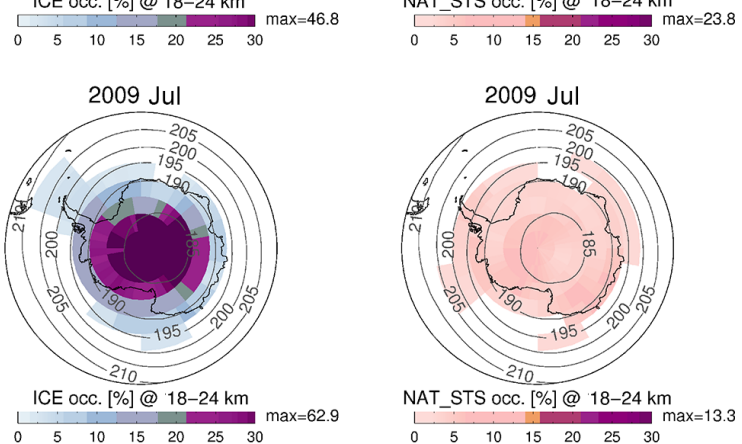

NAT STS occ. [\%] @ 18-24km
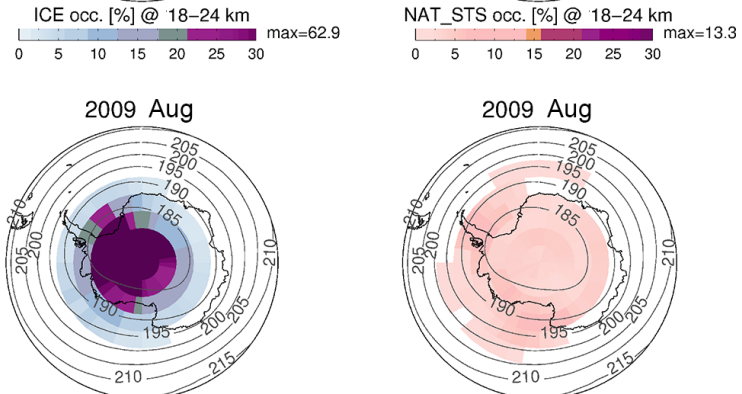

ICE occ. [\%] @ 18-24 km
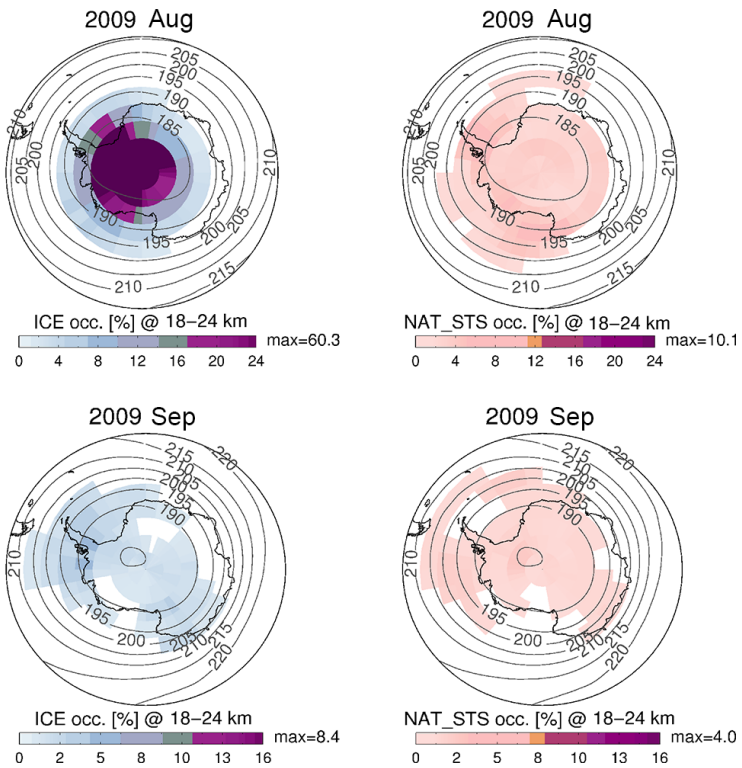

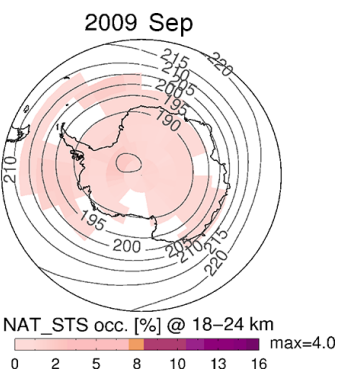

Figure 9. Monthly polar MIPAS PSC type occurrences between 18 and $24 \mathrm{~km}$ for the southern polar winter conditions. Each row shows 1 month (May to September) for 2009 and the respective four main classes STS (orange), NAT (green), ice (blue), and mixed-type class NAT_STS (purple colour code) for the Bayesian classifier. Please note the partly different scaling of the colour code for each month and type. In addition, monthly mean ERAi-based temperature contours at $50 \mathrm{hPa}$ are superimposed. 

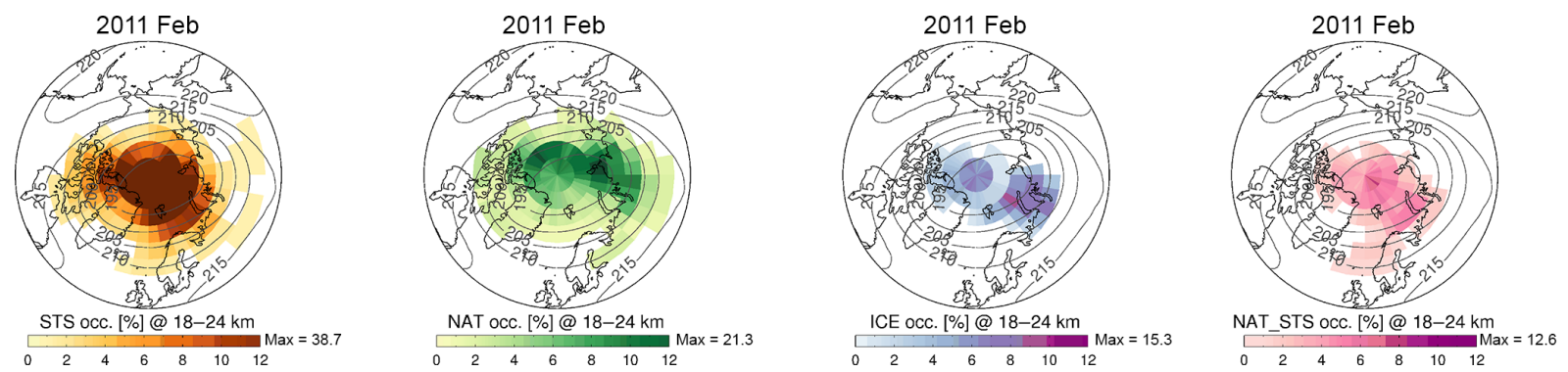

Figure 10. Similar representation of the MIPAS Bayesian classifier PSC classes like in Fig. 9 but for February 2011 only. All PSC classes use the same minimum/maximum values for the colour bar. Maximum values for STS and NAT are significantly larger (38.7 and $21.3 \%$ ) than the maximum colour code value $(12 \%)$.

et al. (2017) for the formation of a NAT belt by MW events downstream of the Antarctic Peninsula. For July (less obvious in June as well), a secondary maximum of NAT occurrence can be seen downstream of the Transatlantic Mountains, an additional hot spot region for MW activity in the Antarctic (Hoffmann et al., 2017). In addition, the June and July occurrence frequencies for ice show an obvious bulge of enhanced activity in the direction of the Antarctic Peninsula, in contrast to the more general concentric distribution around the pole. If these local maxima are directly linked to MW events, a more detailed study is needed, where backward trajectories in conjunction with a microphysical model can establish more profoundly the connection between MW and PSC observations.

Enhanced occurrences for STS are also forming a belt-like structure for June-August. In contrast to the NAT case, this is mainly caused by the synoptic temperatures and also concentric temperature contours in the SH polar vortex, where the quite cold inner polar vortex (south of $70^{\circ} \mathrm{S}$ ) is dominated by ice particles. This results in a belt-like maximum distribution for the STS occurrence frequency. However, in contrast to NAT, STS is not forming a real belt-like structure of clouds on a daily basis of the overall distribution of the PSC classes like in Fig. 2. The mixed-type class NAT_STS shows its maximum occurrence in June and in regions with rather low temperatures, where ice may also be formed. This indicates again the very specific occurrence of this class, where potentially all three types of PSCs are involved.

The NH weekly $A_{\text {PSC }}$ distribution in Fig. 8 already showed the high interannual variability with very distinctive and only sporadic PSC activity for a couple of weeks, with sometimes even no activity over the complete winter. Figure 10 shows only the February 2011 distribution of the four PSC classes of the Bayesian classifier. Due to the generally higher mean and minimum temperatures of the $\mathrm{NH}$ polar vortex, no widespread ice or even no ice at all is observed during typical winter conditions. In this sense, winter 2010/2011 was exceptional; in synoptic-scale regions with temperatures close to and below $T_{\text {ICE }}$, spectra have been frequently observed by MIPAS, creating a large area of ice occurrence with maximum values up to $15 \%$. Ice spectra can be found in the $\mathrm{NH}$ also in other winters, for example, 2009/2010 (von Hobe et al., 2013), but always in a much smaller region (single events) and usually attached to regions where gravity waves may have produced mesoscale temperature fluctuation sufficient to reach the temperature threshold for ice formation (over Greenland, Scandinavian Mountains, or the Ural Mountains; Hoffmann et al., 2017). The Arctic winter 2010/2011 showed also unusually high ice occurrences in the CALIOP data and an exceptionally large ozone depletion over winter and spring close to conditions so far only observed in the SH (Manney et al., 2011; Sinnhuber et al., 2011).

\subsubsection{Temporal $A_{\text {PSC }}$ distributions for each PSC class}

Figure 11 gives an impression of the climatological mean (9 years) daily developments of the area covered by PSCs at a specific altitude (Fig. 11a) and for the specific PSC class of the classifier (Fig. 11b-f). All analysed cloud occurrence frequencies (COFs) for the individual PSC classes are transferred to area-weighted information (e.g. $A_{\mathrm{NAT}}, A_{\mathrm{NAT}}, A_{\mathrm{ICE}}$, and $\left.A_{\text {NAT_STS }}\right)$. The time series for the SH show a number of characteristic and unexpected features.

The maximum in COF for the overall PSC distribution reaches values greater than $56 \%$ with respect to the polar cap region (latitude $>55^{\circ} \mathrm{S}$ ) which is equivalent to an area size of $15.6 \times 10^{6} \mathrm{~km}^{2}$ or the area of a polar cap completely filled with PSCs south of $\sim 70^{\circ} \mathrm{S}$.

There is a significant offset between the mean onset of severe ice, NAT, and STS formation during the early SH winter. In a first phase of 2-3 weeks (mid-May to early June), STS dominates the distribution, followed by the onset of more distinctive ice formation (mid-June), before NAT becomes the dominant PSC type (early July) for most of the time and altitude range for the rest of the winter. This evolution is in line with the typical mean temperature evolution of the vortex, where temperatures usually fall below $T_{\mathrm{ICE}}$ around 1 month after the PSC onset.

The altitude of the maximum in $A_{\text {TYPEi }}$ for each PSC type as well as for the total PSC occurrence is moving contin- 

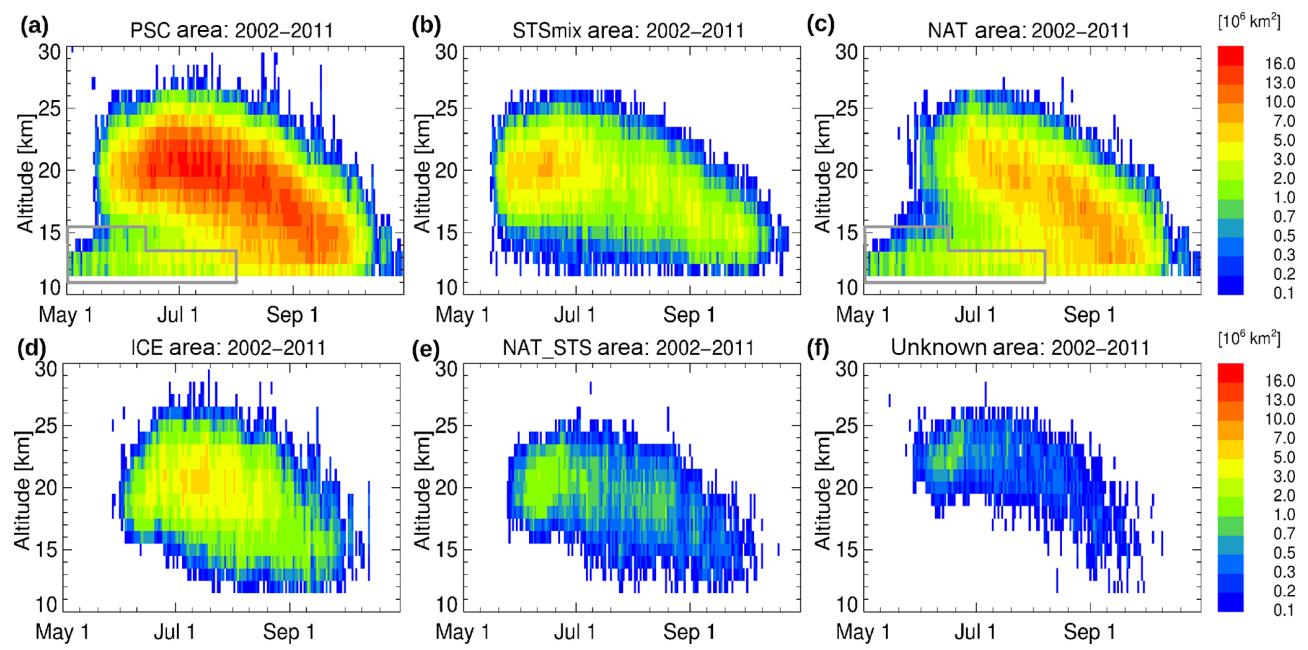

Figure 11. Time series of the 10-year mean (2002, 2003, and 2005-2011) of the daily Antarctic PSC occurrence rates weighted with the area of the applied latitude bins south of $55^{\circ} \mathrm{S}$ in the altitude range $12-30 \mathrm{~km}$. This approach is equivalent to the partition of areal coverage south of $55^{\circ} \mathrm{S}$. An altitude grid of $1 \mathrm{~km}$ with $2 \mathrm{~km}$ grid size is applied. Presented are the overall PSC coverage in in panel (a) and all relevant classes of the Bayesian classifier STSmix, NAT, ICE, NAT_STS, and unknown in panels $(\mathbf{b}-\mathbf{f})$. The grey boxes in the early winter period highlight regions where polar cirrus at the tropopause and potentially some volcanic aerosols introduce a bias in the PSC detection and classification.

uously downward after mid-July, from around 21 down to $13 \mathrm{~km}$ in the direction of the tropopause level. This circumstance follows the typical downward propagation of the vortex cold pool from the mid-stratosphere to the tropopause region for SH polar winter conditions. Dehydration and denitrification processes and the corresponding redistribution of $\mathrm{H}_{2} \mathrm{O}$ and $\mathrm{HNO}_{3}$ over the course of the winter also have an influence on the downward propagation in PSC occurrence.

The mixed-type class NAT_STS shows very similar temporal evolution with height like the STS class, but its major occurrence is limited to the early winter, i.e. the June to early July period, starting just after ice appears for the first time. This suggests that this class of spectra is not only dominated by the radiative properties of STS particles under cold conditions but also with minor and sometimes up to comparable contributions in the spectra by NAT particles with larger radii. Particles with larger NAT radii can be deduced only indirectly, because such events show no significant indication of the typical NAT feature at $820 \mathrm{~cm}^{-1}$ attributed to small NAT particles $(r<3 \mu \mathrm{m})$ (Spang et al., 2005a). Consequently, these events might be related to mixtures of STS, large NAT, and potentially a small volume density of ice particles. Following the same arguments, the class "unknown" may have a similar composition. However, these indications of partitioning of PSC classes are very difficult to quantify and would need further detailed investigation with intensive radiative transport modelling of mixed-type clouds.

There is an unexpected early (beginning of May) and surprising low altitude (close to the tropopause region $\sim 12 \mathrm{~km}$ ) onset of activity for NAT formation in Antarctica. So far it is not completely clear if these events, most likely not related to formation processes of PSCs, are only an artefact of the detection algorithm (e.g. by hypersensitivity) or related to enhanced aerosol load and/or cirrus cloud formation in the UTLS region. Although this is an interesting detail in the observation, the altitude region is obviously not of primary interest for the PSC research of this study. More detailed analyses are planned for future studies with the database.

The large variability for $\mathrm{NH}$ winter conditions, with warm seasons without any PSC sightings and other winters showing synoptic-scaled widespread PSC coverage, results in a very large interannual variability. Although a multi-annual mean like for the SH has only limited representativeness for the NH, Fig. 12 presents a mean climatology of the 10 winter seasons covered by MIPAS. As already pointed out in Sect. 4.1.2, the November-December measurements can be partly contaminated by false PSC detection in the UTLS region (12 up to $16 \mathrm{~km}$, highlighted by the grey box in Fig. 12) due to volcanic eruptions in 2008, 2009, and 2011.

Together with the analyses of the individual winters, the following conclusions are drawn for the $\mathrm{NH}$ observations:

a. STS is always the dominating PSC type for all observed winter periods. This fact suggests that heterogeneous NAT formation at temperatures higher than $T_{\text {ICE }}$ or even higher than $T_{\text {STS }}$, as suggested by Hoyle et al. (2013), might not be the dominating PSC formation mechanism in the NH polar vortex. However, large NAT particles may be classified as STS by the Bayesian classifier, and consequently it is difficult to draw a distinct conclusion from the MIPAS observations. However, usually the temperatures are not low enough in $\mathrm{NH}$ polar vortex for the observation of widespread ice clouds, but this was the case for winter 2010/2011 and to some extent also for winter 2009/2010 (not shown). 

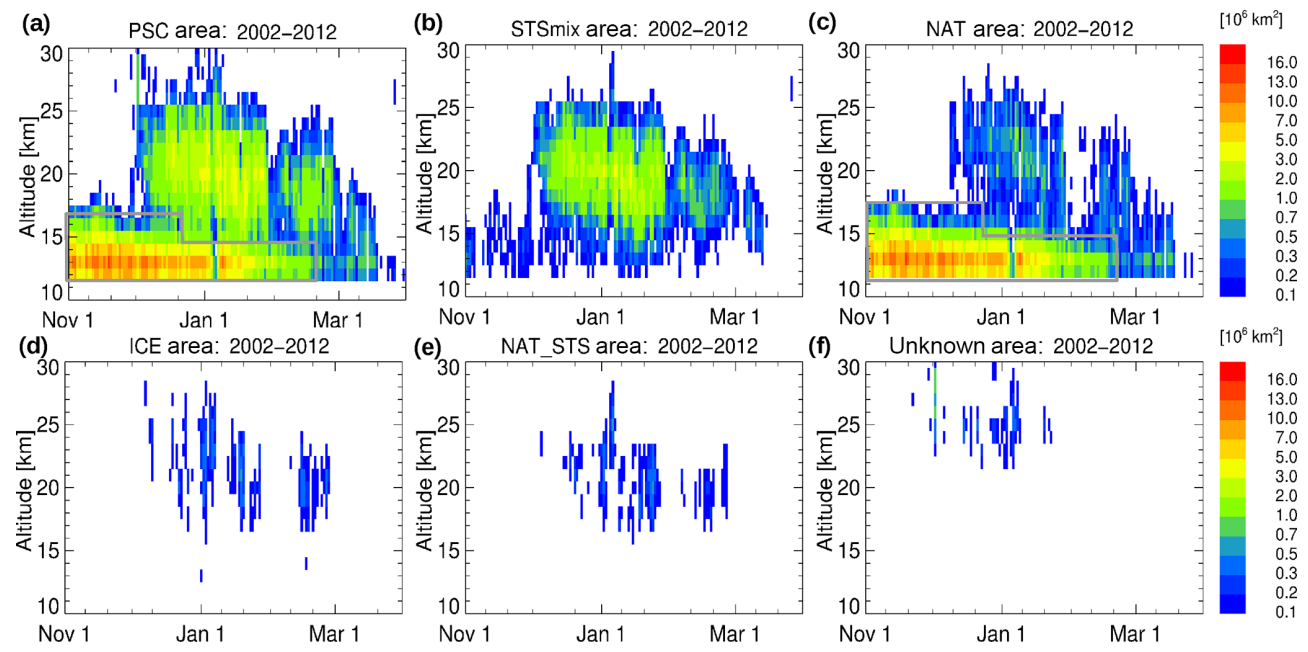

Figure 12. Time series of the northern polar mean 2002/2003 to 2011/2012 winter seasons of the daily Arctic area PSCs of the applied latitude bins north of $55^{\circ} \mathrm{N}$ in the altitude range $12-30 \mathrm{~km}$. This approach is equivalent to the partition of areal coverage north of $55^{\circ} \mathrm{N}$. An altitude grid of $1 \mathrm{~km}$ with $2 \mathrm{~km}$ grid size is applied. Presented are the overall PSC coverage (a) and all relevant classes of the Bayesian classifier (ICE, NAT, STSmix, NAT_STS, and unknown) in panels (b-f). The grey boxes highlight regions where volcanic aerosols in the UTLS have introduced a bias in the PSC detection and classification. The box is based on all winter (November-March) observations in the MIPAS measurement period.

b. Ice PSCs are a rare observation but have been observed sporadically with the earliest events in the first half of December and the latest by the end of February, depending on the individual winter.

c. The de-nitrification in the middle stratosphere and renitrification in the lower stratosphere combined with the subsidence with height of the cold pool over the winter becomes also obvious in the NAT distribution but less pronounced than in the $\mathrm{SH}$.

d. The maximum altitude of PSC occurrences is higher in the $\mathrm{NH}$ than in the $\mathrm{SH}(1-2 \mathrm{~km}$ and reaches CTHs up to $28-30 \mathrm{~km}$ ). This difference may be caused by a signalto-noise ratio issue for altitudes of $\sim 30 \mathrm{~km}$ and above (Spang et al., 2004, 2012). At these altitudes, the cold stratospheric temperatures yield only very weak radiance signals in the atmospheric window region (close to the detector noise level) used in the cloud index approach. Consequently, cloud index profiles start to get noisy above $\sim 30 \mathrm{~km}$ and cloud detection becomes more difficult. This effect is stronger in the $\mathrm{SH}$ than in the $\mathrm{NH}$, with a larger and colder polar vortex in the SH. This may cause an underestimation in PSC occurrence at $\sim 30 \mathrm{~km}$ in the $\mathrm{SH}$.

e. The relatively high NAT occurrence frequency in November in the Arctic in the UTLS region is a prolonged feature and a perplexing observation. The phenomenon is definitely related to the enhanced volcanic activity in the time frame of the analysis, where four significant eruptions at midlatitudes had a severe im- print on the UTLS aerosol load in the NH (see also Sect. 4.1.2). The aerosols of volcanic origin have similar radiative properties to NAT particles, because no other PSC type shows such a significant occurrence enhancement in the UTLS for the three seasons contaminated by volcanic eruptions. Why the classifier selects for these altitudes for most of the cloud events the PSC class NAT instead of the more sulfuric-acid-related STS class is still an open question. These events occur mainly in November and at altitudes near the tropopause where the temperatures are clearly too high to form PSCs (both NAT and STS). Consequently, these false PSC detections are easy to exclude from further analysis by simple altitude and temperature thresholds.

\subsection{3 $A_{\mathrm{PSC}}$ based on reanalysis temperature data for the SH winter 2010}

Generally, there are difficulties in finding a sophisticated approach to retrieve characteristic and comparable parameters from the very different information of remote sensing measurements and the model output of CCMs and CTMs for PSC particles. Some studies use the complex way to retrieve the measurement quantity, e.g. attenuated backscatter signals of lidars or radiances for passive IR measurements, from the model parameters with a corresponding forward model, for example, Engel et al. (2013) for CALIOP or Höpfner et al. (2006b) for MIPAS measurements. For this kind of approach, it would be also necessary to sample the model with the asynoptic measurement net of the instrument and for MIPAS to take the effect of limb path integration into account 

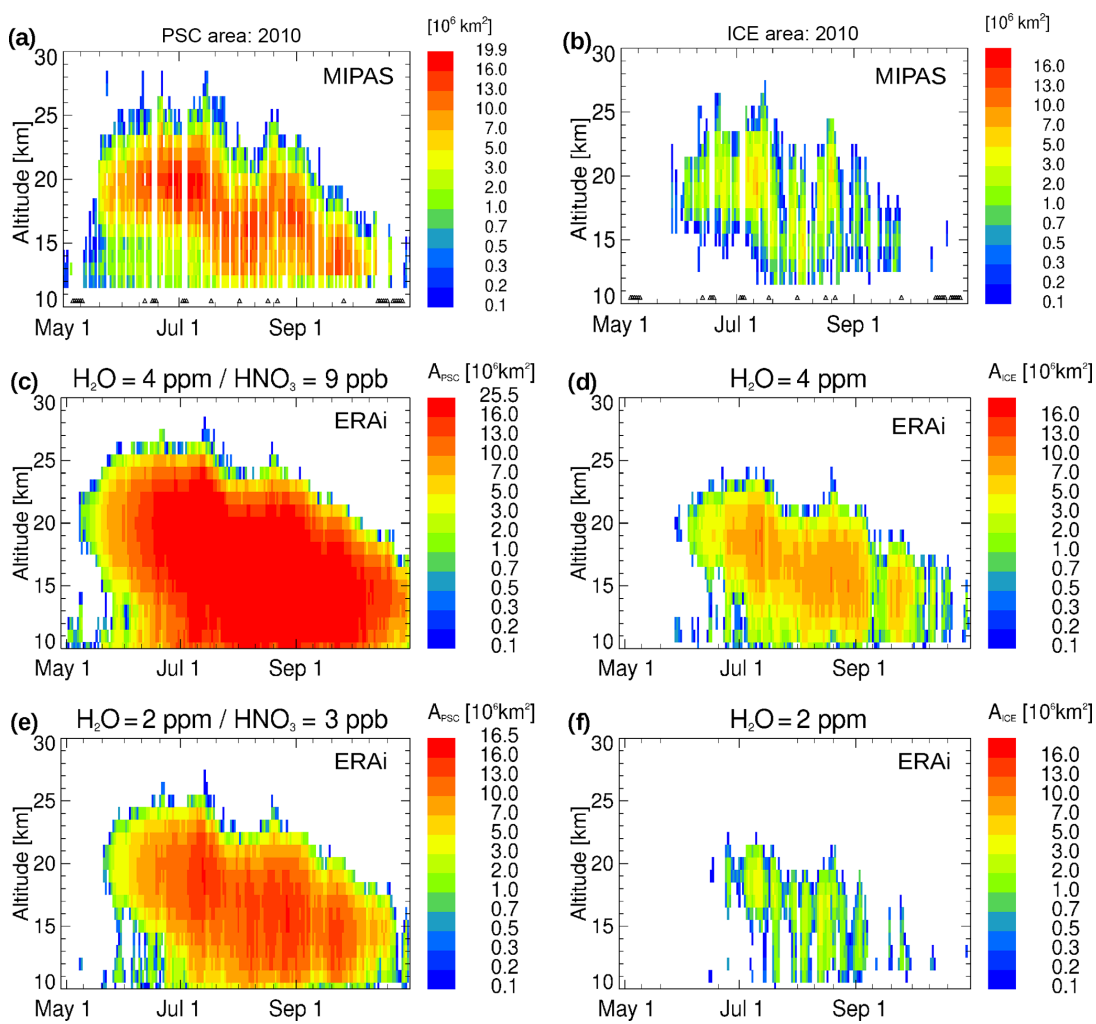

Figure 13. Time series of the daily Antarctic area of PSCs $\left(A_{\mathrm{PSC}}\right)$ including all PSC classes and the area of the class ICE only $\left(A_{\mathrm{ICE}}\right)$ for the year 2010 retrieved from MIPAS spectra (a, b; see also Fig. 11). This is compared with the standard calculation of PSC areas based on ERAi temperature data with constant stratospheric water vapour and $\mathrm{HNO}_{3}$ values (c-f). Results of two setups are presented for $\mathrm{H}_{2} \mathrm{O}$ of 4 and 2 ppmv, respectively, in panels (c, d) and (e, f), and for $\mathrm{HNO}_{3}$ of 9 and 3 ppbv, respectively, (c, e) to illustrate the usually observed effects of denitrification and dehydration in the polar vortex over the winter.

(e.g. Spang et al., 2012). However, this very expensive approach, with large numerical costs and extensive effort for implementation and validation of the methodology, is only practical for more detailed case studies of a subset of the data.

Figure 13 presents the time series of the overall MIPAS PSC and ice class (top row) coverage for the Southern Hemisphere together with the results of a computation of $A_{\mathrm{PSC}}$ and $A_{\text {ICE }}$ based on temperature data of a meteorological reanalysis dataset (ERA-Interim). The comparison illustrates the potential benefit of the new climatology for validation and consistency purposes of CTMs and CCMs. A more detailed comparison with the Chemical Lagrangian Model for the Stratosphere (CLaMS) including a sophisticated microphysical model (Grooß et al., 2014) is presented in a parallel study by Tritscher et al. (2018).

For the ERAi-based dataset, a grid box is characterised as cloudy if the temperature at the grid point falls below the PSC existence temperature $\left(T_{\mathrm{NAT}}\right)$. If the temperature falls below the existence temperature for ice ( $\left.T_{\mathrm{ICE}}\right)$, the grid box is additionally marked as an ice cloud. $T_{\mathrm{NAT}}$ and $T_{\mathrm{ICE}}$ are functions of pressure, $\mathrm{H}_{2} \mathrm{O}$, and $\mathrm{HNO}_{3}$ abundance (Hanson and Mauersberger, 1988; Marti and Mauersberger, 1993). Finally, $A_{\mathrm{PSC}}$ and $A_{\mathrm{ICE}}$ are computed with an analogous approach to the MIPAS data by simply using the grid point of ERAi (longitude, latitude, and geopotential height) instead of the tangent height locations of MIPAS. Constant water vapour and nitric acid concentrations are assumed in the stratosphere for the definition of the corresponding existence temperatures of NAT and ice PSCs. For better representation of the dehydration and denitrification process over the winter, we computed the distributions for two setups: the high case (Fig. 13c and d), with high water and nitric acid concentration typical for early winter (4 ppmv and $9 \mathrm{ppbv}$, respectively), and the low case (Fig. 13e and f), with mixing ratios more typical for late winter after severe denitrification and dehydration took place ( 2 ppmv and 3 ppbv). High and low cases together represent a realistic min/max scenario of the $A_{\mathrm{PSC}}$ and $A_{\mathrm{ICE}}$ distribution based on reanalysis temperatures. More realistic $\mathrm{HNO}_{3}$ and $\mathrm{H}_{2} \mathrm{O}$ values could be deduced from, for example, satellite measurements.

The MIPAS measurements show a significant break in PSC occurrence activity during the end of July/early August period. This is most obvious in $A_{\mathrm{ICE}}$, but also the maximum $A_{\text {PSC }}$ altitude range (reddish area) is shifting up and down during this phase of the winter. The ERAi analyses show a similar but less pronounced effect, indicating a warming 

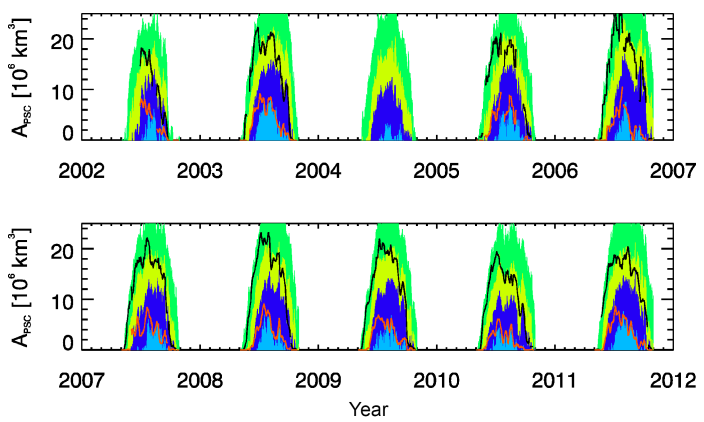

Figure 14. SH interseasonal variation in $A_{\mathrm{PSCmax}}$ between 15 and $30 \mathrm{~km}$ with the black line for MIPAS (7-day running mean), green and light green coloured areas retrieved from ERA-Interim daily temperature fields and fixed $\mathrm{H}_{2} \mathrm{O} / \mathrm{HNO}_{3}$ profile for high case and low case (see Sect. 4.2.3) and $A_{\text {ICEmax }}$ with a red line for MIPAS, blue and light blue bars for ERA-Interim high case and low case, respectively. In the Antarctic polar winter of 2004, MIPAS measurements are not available.

event for parts of the polar vortex and consequently less PSC occurrence, especially for ice. In addition, the comparison indicates an overestimation of $A_{\mathrm{PSC}}$ by the simple temperaturebased estimate of these quantities for most of the winter. Larger $A_{\mathrm{PSC}}$ values over an extended altitude range are predicted even in the ERAi low case. For $A_{\text {ICE }}$, a mixture of the ERAi high case and low case could possibly fit the vertical distribution quite well, but the high case with a realistic value of 4 ppmv for early to mid-winter conditions is creating a far too-large $A_{\text {ICE }}$ in the ERAi analysis compared to the MIPAS measurements.

a. A potential reason could be potential cold bias in the reanalysis data. This would be in contradiction to a very recent study of reanalysis temperature data which shows a slight warm bias for the polar lower stratosphere for ERAi (Lambert and Santee, 2018).

b. Another potential reason could be a lack of sensitivity of MIPAS to detect all forms of ice clouds. For example, optically very thin clouds or clouds only partly in the relatively broad vertical FOV of MIPAS (3-4 km) might be missed or misclassified. However, this explanation seems unlikely due to the extremely high detection sensitivity of an IR limb sounder with respect to ice clouds and usually strong cloud signals even when ice is covering only a part of the measurement volume (Spang et al., 2012, 2015, 2016).

c. Yet another potential reason could be that the simple temperature-based method is not accurate enough to describe the occurrence of PSCs with respect to vertical distribution and temporal evolution over the winter.

The temperature-based $A_{\text {PSC }}$ can only be a rough proxy for the real PSC coverage because detailed microphysical modelling needs to be considered for an adequate estimate of the
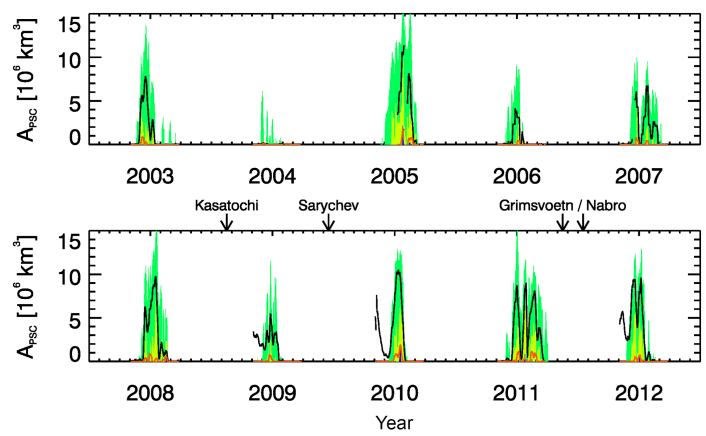

Figure 15. NH interseasonal variation of $A_{\mathrm{PSCmax}}$ and $A_{\text {ICEmax }}$ based on ERA-Interim and MIPAS (see Fig. 14). In the lower panel, dates of NH volcanic eruptions with stratospheric aerosol burden are superimposed to highlight the link to the artificial $A_{\text {PSC }}$ enhancements in November 2008, 2009, and 2011 and which are not caused by PSCs. Please note that data before 1 November are not considered in the analysis.

PSC type coverage. Studies by Engel et al. (2013) and Grooß et al. (2014) showed that detailed microphysical modelling of PSCs is essential to get an adequate agreement in the PSC coverage and types' distributions along segments of CALIOP orbits covered by PSCs.

Nevertheless, the comparison shows that SH PSC occurrence can show a substantial interannual variability in the temporal evolution of the PSC type occurrence for particular winters. The major stratospheric warming event in 2002 with a split of the vortex and the ozone hole has been reported already above and is highlighted in the literature as a unique event (e.g. Newman and Nash, 2005). However, also the winter of 2010 shows an enhanced variability in the PSC distribution that is related to a less pronounced warming event in the stratosphere.

\subsubsection{Interseasonal time series of PSC and ice coverage}

The results of Sect. 4.2.3 and 4.1.2 already show the benefit a climatology of PSC types can provide for validation purposes of models and for complementary measurements (ground-based, air-, and spaceborne sensors) as well as reanalyses. Figures 14 and 15 present a comparison of the complete time series of $A_{\mathrm{PSCmax}}$ and $A_{\text {ICEmax }}$ retrieved from the MIPAS PSC climatology with the ERAi-based maximum $T_{\mathrm{NAT}} / T_{\mathrm{ICE}}$ area for the high-case/low-case scenarios of $\mathrm{H}_{2} \mathrm{O} / \mathrm{HNO}_{3}$ concentrations (see Sect. 4.2.3). The MIPAS time series are smoothed by taking a 7-day running mean. Data gaps in the time series due to changing measurement modes and restricted operation times of the instrument in 2005 and 2006 make this smoothing essential to obtain a continuous time series.

The comparison shows a number of characteristic features:

a. The onset of PSC formation is remarkably well reproduced for the more realistic ERAi high case. Nearly all 
winter seasons in the $\mathrm{SH}$ show small offsets in the range of a couple of days.

b. The NH shows larger differences for the onset, indicating difficulties in the prediction of PSC occurrence by the simple methodology for the more variable conditions of NH polar stratosphere.

c. Even the SH onsets of ice formation agree reasonably well for the more realistic ERAi high case.

d. The end of the PSC seasons, which should be better compared to the ERAi low case, show a less good correspondence (earlier interruption of PSC activity in the observations). Re-nitrification and rehydration at lower altitudes will modify the trace gas distributions but this is not considered in the simple high-case/low-case approach.

e. The absolute values of $A_{\text {PSCmax }}$ and $A_{\text {ICEmax }}$ compare remarkably well for the low-case scenario, whereas the high case shows overestimation for the $\mathrm{SH}$ and $\mathrm{NH}$ conditions. More reliable information on the spatial and temporal evolution of $\mathrm{HNO}_{3}$ and $\mathrm{H}_{2} \mathrm{O}$ over the winter, for example, from satellite measurements, could improve absolute values of the ERAi temperature-based parameters. CTM and CCM deliver simultaneous and consistent trace gas and temperature information. Comparison with the MIPAS time series might help to improve and consolidate the PSC parameters of the models.

f. Volcanic eruptions have produced artificial PSC coverage in the $\mathrm{NH}$ for November 2008, 2009, and 2011 by enhancing the aerosol load in the UTLS (see also Sect. 4.1.2). Especially the eruption of Sarychev in June 2009 created a long-lasting aerosol cloud covering large parts of the NH up to the pole (Wu et al., 2017).

The results of the comparison between the temporally smoothed and vertically integrated maximum area of PSC coverage of MIPAS with the simple temperature-based PSC proxies show that the overall winter evolution can be modelled reasonably well. However, more detailed and less smoothed analyses for individual winters of $A_{\text {TYPE }}$ show significant differences (see Fig. 13). However, a similar approach applied to the output parameters of global models could be a valuable tool to quantify the quality of PSCrelated processes in CCMs and GCMs.

Comparisons of the hemispheric distribution of PSC classes on a daily basis like in Fig. 2 frequently show discrepancies with respect to the superimposed contours of temperature thresholds, although the comparison of the overall integrated information of $A_{\mathrm{PSCmax}}$ or $A_{\text {ICEmax }}$ looks promising. The discrepancies highlight the difficulties to predict the detailed temporal and spatial distribution of the PSC types correctly, in situations when additional and more detailed modelling of the microphysical and dynamical processes (e.g. heterogeneous/homogenous nucleation, mountain waves) is becoming essential.

\section{Summary and conclusions}

The unique dataset of 10 years of MIPAS limb IR spectra has been analysed with a Bayesian classifier to distinguish various PSC classes (Spang et al., 2016). We detected cloudy and classified spectra of $10 \mathrm{NH}$ and nine SH polar winters. The classification delivers the main classes STS, NAT, and ice but also less stringent classifiable types of spectra described with NAT_STS for a mixed-type class and spectra which cannot reliably classified at all (the so-called "unknown" class).

The classification is guided by the characteristic spectral shape in the emission spectra of the different PSC particles and is sensitive to specific features or characteristic gradients over the selected atmospheric window regions of the IR spectrum. The measured radiation is frequently dominated by the spectral shape of only one particle type but in the case of mixtures this type is not necessarily the particle type with the largest volume density or area density along the line of sight of the instrument. Due to the long limb path of the instrument, the detection sensitivity is excellent and in the range of spaceborne lidars. The temporal and vertical evolution of area covered by all PSC classes ( $A_{\text {PSC }}$ ) and specifically ice ( $\left.A_{\text {ICE }}\right)$ shows a good correspondence with the new version 2 dataset of PSC detection and classification from the CALIOP lidar measurements onboard the CALIPSO satellite.

Various comparisons and applications of the MIPAS climatology have been presented in this study and the main results will be highlighted as follows.

Frequency distributions of $T-T_{\mathrm{ICE}}$ for the main PSC classes of the Bayesian classifier using ERAi coincident temperatures show reliable distributions with respect to the current understanding of PSC particle formation. The mixedtype class NAT_STS shows a striking tendency for quite cold temperatures and is mainly observed in the first half of the $\mathrm{SH}$ winter. This may point to a special mixture of NAT and STS with some potentially weak contributions of ice.

The monthly horizontal distribution in the PSC class occurrence frequencies shows pronounced geographical regions with enhanced PSC activity for the SH. Some of these regions show for specific months of the multi-annual mean a link with regions well known for strong MW activity (e.g. a belt downstream of the Antarctic Peninsula with enhanced NAT occurrence, and a local maximum in the ice occurrence over the Antarctic Peninsula). Ice is usually dominating the inner part of the polar vortex and most for July and August consistent with the development of the stratospheric mean temperature distribution. 
$A_{\text {PSC }}$ (and $A_{\text {TYPEi }}$ ) retrieved from the classifier results can be used as a quantitative measure for the daily evolution and strength of the PSC distribution over winter. The parameters are useful for comparison with model-based or simple analysed temperature-based $A_{\text {TYPEi }}$ time series for validation purposes. In a second step, the conclusions may allow the partially simple microphysical schemes applied in CCMs and CTMs to be improved and optimised.

A comparison of the MIPAS-based and simple temperature-based $A_{\mathrm{PSC}}$ and $A_{\mathrm{TYPEi}}$ distributions shows that models with a simple temperature threshold parameterisation of the PSC coverage require a good representation of the temporal and spatial water vapour and nitric acid distribution over the winter to adequately reproduce the "real" measured $A_{\text {TYPEi }}$ distribution.

The MIPAS PSC climatology (2002-2012) and the 11year time series of CALIOP measurements (2006-present) constitute an unprecedented and comprehensive overview of the formation processes and temporal development of PSCs in the $\mathrm{NH}$ and $\mathrm{SH}$ winter polar vortices on vortex-wide scales. Together with the MLS trace gas measurements on EOS-Aura, process studies can now address a number of still imprecisely understood processes (formation of large NAT particles, heterogeneous and/or homogeneous nucleation, types of condensation nuclei fostering PSC formation). Moreover, combining the PSC information from MIPAS and CALIOP with the measurements of key chlorine-containing species from MLS and MIPAS will furthermore allow to evaluate the impact of different PSC types on the chlorine chemistry.

These improvements will contribute to a better and more reliable prediction of the future polar ozone in a changing climate. Upcoming results of the currently widespread investigations for improvements and more realistic handling of the PSC schemes in state-of-the-art CTMs and CCMs will need comprehensive datasets for consistency tests and validation purposes. Currently, there is no comparable combination of satellite instruments part of the strategic programs of the international space agencies. Consequently, these datasets will have to act as a reference climatology for the hemispheric PSC coverage and type distribution for a very long time.

Data availability. The ERA-Interim dataset has been made available for download by the European Centre for Medium-Range Weather Forecasts (ECMWF) (Dee et al., 2011). The meteorological data such as temperature, pressure, and geopotential height are interpolated to the measurement location of MIPAS (tangent point) and are part of the climatology data product.

The MIPAS PSC climatology consists of all measurement points of the middle stratosphere down to the lowest tangent point of a single measurement profile for the time period of June 2002 to March 2012. The dataset is available in user-friendly NetCDF format (https://www.unidata.ucar.edu/software/netcdf, last access: 19 September 2017). In addition to the geospatial information (altitude, longitude, and latitude), the parameter class includes the classifica- tions result $[-1, \ldots, 7]$ for the classes [no-cloud, unknown, Ice, NAT, STS, ICE_NAT, NAT_STS, and ICE_STS]. The corresponding three product probabilities $\left(P_{\mathrm{ICE}}, P_{\mathrm{NAT}}\right.$, and $\left.P_{\mathrm{STS}}\right)$ used in the Bayesian classifier are listed for each cloudy spectrum and can be used as a quality check of the classification approach.

The dataset is registered by r3edata.org (http://www.re3data.org/ repository/r3d100012449) with a corresponding digital object identifier (https://doi.org/10.17616/R3BN26; r3edata.org, 2017) and is available for download for the scientific community via http: //datapub.fz-juelich.de/slcs/mipas/psc (last access: 9 April 2018; daily files for May-October for the SH and November-March for the NH PSC seasons). Preview images are available on a daily basis and at a number of potential temperature intervals. The presented secondary data products like $A_{\mathrm{PSC}}$ or PSC occurrence frequencies can be obtained on request from the first author.

Competing interests. The authors declare that they have no conflict of interest.

Acknowledgements. We gratefully acknowledge ECMWF, ESA, and NASA for the preparation of the ERA-Interim dataset, the MIPAS Level 1 data, and the CALIOP PSC data, respectively. Reinhold Spang thanks Sabine Griessbach (FZJ) for discussions and references on the topic of the volcanic aerosol load of the stratosphere. Ines Tritscher was funded by the Deutsche Forschungsgemeinschaft (DFG) under project number 310479827. Finally, the authors like to thank the International Space Science Institute (ISSI) and Stratosphere-troposphere Processes And their Role in Climate (SPARC) for supporting the activities on the compilation of a PSC climatological dataset.

The article processing charges for this open-access publication were covered by a Research Centre of the Helmholtz Association.

Edited by: Farahnaz Khosrawi

Reviewed by: Ellis Remsberg and two anonymous referees

\section{References}

Achtert, P. and Tesche, M.: Assessing lidar-based classification schemes for polar stratospheric clouds based on 16 years of measurements at Esrange, Sweden, J. Geophys. Res., 119, 13861405, https://doi.org/10.1002/2013JD020355, 2014.

Allen, D. R., Bevilacqua, R. M., Nedoluha, G. E., Randall, C. E., and Manney, G. L.: Unusual stratospheric transport and mixing during the 2002 Antarctic winter, Geophys. Res. Lett., 30, 1599, https://doi.org/10.1029/2003GL017117, 2003.

Arnone, E., Castelli, E., Papandrea, E., Carlotti, M., and Dinelli, B. M.: Extreme ozone depletion in the 2010-2011 Arctic winter stratosphere as observed by MIPAS/ENVISAT using a 2D tomographic approach, Atmos. Chem. Phys., 12, 9149-9165, https://doi.org/10.5194/acp-12-9149-2012, 2012.

Butler, A. H., Sjoberg, J. P., Seidel, D. J., and Rosenlof, K. H.: A sudden stratospheric warming compendium, Earth Syst. Sci. Data, 9, 63-76, https://doi.org/10.5194/essd-9-63-2017, 2017. 
Carslaw, K. S., Luo, B., and Peter, T.: An analytic expression for the composition of aqueous $\mathrm{HNO}_{3}-\mathrm{H}_{2} \mathrm{SO}_{4}$ stratospheric aerosols including gas phase removal of $\mathrm{HNO}_{3}$, Geophys. Res. Lett., 22, 1877-1880, https://doi.org/10.1029/95GL01668, 1995.

Carslaw, K. S., Peter, T., and Müller, R.: Uncertainties in reactive uptake coefficients for solid stratospheric particles - 2. Effect on ozone depletion, Geophys. Res. Lett., 24, 1747-1750, https://doi.org/10.1029/97GL01684, 1997.

Carslaw, K. S., Wirth, M., Tsias, A., Luo, B. P., Dörnbrack, A., Leutbecher, M., Volkert, H., Renger, W., Bacmeister, J. T., Reimer, E., and Peter, T.: Increased stratospheric ozone depletion due to mountain-induced atmospheric waves, Nature, 391, 675-678, https://doi.org/10.1038/35589, 1998.

Dee, D. P., Uppala, S. M., Simmons, A. J., Berrisford, P., Poli, P., Kobayashi, S., Andrae, U., Balmaseda, M. A., Balsamo, G., Bauer, P., Bechtold, P., Beljaars, A. C. M., van de Berg, L., Bidlot, J., Bormann, N., Delsol, C., Dragani, R., Fuentes, M., Geer, A. J., Haimberger, L., Healy, S. B., Hersbach, H., Holm, E. V., Isaksen, L., Kallberg, P., Koehler, M., Matricardi, M., McNally, A. P., Monge-Sanz, B. M., Morcrette, J. J., Park, B. K., Peubey, C., de Rosnay, P., Tavolato, C., Thepaut, J. N., and Vitart, F.: The ERA-Interim reanalysis: configuration and performance of the data assimilation system, Q. J. Roy. Meteor. Soc., 137, 553-597, https://doi.org/10.1002/qj.828, 2011.

De Laat, A. T. J. and van Weele, M.: The 2010 Antarctic ozone hole: Observed reduction in ozone destruction by minor sudden stratospheric warmings, Scientific Reports, 1, 38, https://doi.org/10.1038/srep00038, 2011.

Di Liberto, L., Cairo, F., Fierli, F., Di Donfrancesco, G., Viterbini, M., Deshler, T., and Snels, M.: Observation of polar stratospheric clouds over McMurdo $\left(77.85^{\circ} \mathrm{S}, 166.67^{\circ} \mathrm{E}\right)$ (2006-2010), J. Geophys. Res.-Atmos., 119, 5528-5541, https://doi.org/10.1002/2013JD019892, 2014.

Dörnbrack, A., Birner, T., Fix, A., Flentje, H., Meister, A., Schmid, H., Browell, E. V., and Mahoney, M. J.: Evidence for inertia gravity waves forming polar stratospheric clouds over Scandinavia, J. Geophys. Res., 107, 8287, https://doi.org/10.1029/2001JD000452, 2002.

Drdla, K. and Müller, R.: Temperature thresholds for chlorine activation and ozone loss in the polar stratosphere, Ann. Geophys., 30, 1055-1073, https://doi.org/10.5194/angeo-30-10552012, 2012.

Eckermann, S. D., Hoffmann, L., Höpfner, M., Wu, D. L., and Alexander, M. J.: Antarctic NAT PSC belt of June 2003: Observational validation of the mountain wave seeding hypothesis, Geophys. Res. Lett., 36, L02807, https://doi.org/10.1029/2008GL036629, 2009.

Engel, I., Luo, B. P., Pitts, M. C., Poole, L. R., Hoyle, C. R., Grooß, J.-U., Dörnbrack, A., and Peter, T.: Heterogeneous formation of polar stratospheric clouds - Part 2: Nucleation of ice on synoptic scales, Atmos. Chem. Phys., 13, 10769-10785, https://doi.org/10.5194/acp-13-10769-2013, 2013.

Eyring, V., Arblaster, J. M., Cionni, I., Sedlácek, J., Perlwitz, J., Young, P. J., Bekki, S., Bergmann, D., Cameron-Smith, P., Collins, W. J., Faluvegi, G., Gottschaldt, K.-D., Horowitz, L. W., Kinnison, D. E., Lamarque, J.-F., Marsh, D. R., Saint-Martin, D., Shindell, D. T., Sudo, K., Szopa, S., and Watanabe, S.: Long-term ozone changes and associated climate impacts in
CMIP5 simulations, J. Geophys. Res.-Atmos., 118, 5029-5060, https://doi.org/10.1002/jgrd.50316, 2013.

Fahey, D. W., Gao, R. S., Carslaw, K. S., Kettleborough, J., Popp, P. J., Northway, M. J., Holecek, J. C., Ciciora, S. C., McLaughlin, R. J., Thompson, T. L., Winkler, R. H., Baumgardner, D. G., Gandrud, B., Wennberg, P. O., Dhaniyala, S., McKinley, K., Peter, T., Salawitch, R. J., Bui, T. P., Elkins, J. W., Webster, C. R., Atlas, E. L., Jost, H., Wilson, J. C., Herman, R. L., Kleinböhl, A., and von König, M.: The detection of large $\mathrm{HNO}_{3}$ containing particles in the winter Arctic stratosphere, Science, 291, 1026-1031, 2001.

Fernandez, R. P., Kinnison, D. E., Lamarque, J.-F., Tilmes, S., and Saiz-Lopez, A.: Impact of biogenic very short-lived bromine on the Antarctic ozone hole during the 21st century, Atmos. Chem. Phys., 17, 1673-1688, https://doi.org/10.5194/acp-171673-2017, 2017.

Fischer, H., Birk, M., Blom, C., Carli, B., Carlotti, M., von Clarmann, T., Delbouille, L., Dudhia, A., Ehhalt, D., Endemann, M., Flaud, J. M., Gessner, R., Kleinert, A., Koopman, R., Langen, J., López-Puertas, M., Mosner, P., Nett, H., Oelhaf, H., Perron, G., Remedios, J., Ridolfi, M., Stiller, G., and Zander, R.: MIPAS: an instrument for atmospheric and climate research, Atmos. Chem. Phys., 8, 2151-2188, https://doi.org/10.5194/acp-8-21512008, 2008.

Fromm, M., Alfred, J., and Pitts, M.: A unified, long-term, highlatitude stratospheric aerosol and cloud database using SAM II, SAGE II, and POAM II/III data: Algorithm description, database definition, and climatology, J. Geophys. Res., 108, 4366, https://doi.org/10.1029/2002JD002772, 2003.

Griessbach, S., Hoffmann, L., Spang, R., von Hobe, M., Müller, R., and Riese, M.: Infrared limb emission measurements of aerosol in the troposphere and stratosphere, Atmos. Meas. Tech., 9, 4399-4423, https://doi.org/10.5194/amt-9-4399-2016, 2016.

Grooß, J.-U., Engel, I., Borrmann, S., Frey, W., Günther, G., Hoyle, C. R., Kivi, R., Luo, B. P., Molleker, S., Peter, T., Pitts, M. C., Schlager, H., Stiller, G., Vömel, H., Walker, K. A., and Müller, R.: Nitric acid trihydrate nucleation and denitrification in the Arctic stratosphere, Atmos. Chem. Phys., 14, 1055-1073, https://doi.org/10.5194/acp-14-1055-2014, 2014.

Günther, A., Höpfner, M., Sinnhuber, B.-M., Griessbach, S., Deshler, T., von Clarmann, T., and Stiller, G.: MIPAS observations of volcanic sulfate aerosol and sulfur dioxide in the stratosphere, Atmos. Chem. Phys., 18, 1217-1239, https://doi.org/10.5194/acp-18-1217-2018, 2018.

Hanson, D. and Mauersberger, K.: Laboratory studies of the nitric acid trihydrate: implications for the south polar stratosphere, Geophys. Res. Lett., 15, 855-858, 1988.

Hervig, M. E., Carslaw, K. S., Peter, T., Deshler, T., Gordley, L. L., Redaelli, G., Biermann, U., and Russell, J. M.: Polar stratospheric clouds due to vapor enhancement: HALOE observations of the Antarctic vortex in 1993, J. Geophys. Res., 102, 2818528193, 1997.

Hoffmann, L., Spang, R., Orr, A., Alexander, M. J., Holt, L. A., and Stein, O.: A decadal satellite record of gravity wave activity in the lower stratosphere to study polar stratospheric cloud formation, Atmos. Chem. Phys., 17, 2901-2920, https://doi.org/10.5194/acp-17-2901-2017, 2017.

Höpfner, M., Oelhaf, H., Wetzel, G., Friedl-Vallon, F., Kleinert,, A., Lengel, A., Maucher, G., Nordmeyer, H., Glatthor, N., Stiller, 
G. P., von Clarmann, T., Fischer, H., Kröger, C., and Deshler, T.: Evidence of scattering of tropospheric radiation by PSCs in mid-IR limb emission spectra: MIPAS-B observations and KOPRA simulations, Geophys. Res. Lett., 29, 119-1-119-4, https://doi.org/10.1029/2001GL014443, 2002.

Höpfner, M., Luo, B. P., Massoli, P., Cairo, F., Spang, R., Snels, M., Di Donfrancesco, G., Stiller, G., von Clarmann, T., Fischer, H., and Biermann, U.: Spectroscopic evidence for NAT, STS, and ice in MIPAS infrared limb emission measurements of polar stratospheric clouds, Atmos. Chem. Phys., 6, 1201-1219, https://doi.org/10.5194/acp-6-1201-2006, 2006a.

Höpfner, M., Larsen, N., Spang, R., Luo, B. P., Ma, J., Svendsen, S. H., Eckermann, S. D., Knudsen, B., Massoli, P., Cairo, F., Stiller, G., v. Clarmann, T., and Fischer, H.: MIPAS detects Antarctic stratospheric belt of NAT PSCs caused by mountain waves, Atmos. Chem. Phys., 6, 1221-1230, https://doi.org/10.5194/acp-61221-2006, 2006b.

Hoyle, C. R., Engel, I., Luo, B.P., Pitts, M. C., Poole, L.R., Grooß, J.-U., and Peter, T.: Heterogeneous formation of polar stratospheric clouds - Part 1: Nucleation of nitric acid trihydrate (NAT), Atmos. Chem. Phys., 13, 9577-9595, https://doi.org/10.5194/acp-13-9577-2013, 2013.

Hurley, J., Dudhia, A., and Grainger, R. G.: Retrieval of macrophysical cloud parameters from MIPAS: algorithm description, Atmos. Meas. Tech., 4, 683-704, https://doi.org/10.5194/amt-4683-2011, 2011.

Kirner, O., Müller, R., Ruhnke, R., and Fischer, H.: Contribution of liquid, NAT and ice particles to chlorine activation and ozone depletion in Antarctic winter and spring, Atmos. Chem. Phys., 15, 2019-2030, https://doi.org/10.5194/acp-15-2019-2015, 2015.

Lambert, A. and Santee, M. L.: Accuracy and precision of polar lower stratospheric temperatures from reanalyses evaluated from A-Train CALIOP and MLS, COSMIC GPS RO, and the equilibrium thermodynamics of supercooled ternary solutions and ice clouds, Atmos. Chem. Phys., 18, 1945-1975, https://doi.org/10.5194/acp-18-1945-2018, 2018.

Lambert, A., Santee, M. L., Wu, D. L., and Chae, J. H.: Atrain CALIOP and MLS observations of early winter Antarctic polar stratospheric clouds and nitric acid in 2008, Atmos. Chem. Phys., 12, 2899-2931, https://doi.org/10.5194/acp-122899-2012, 2012.

Lowe, D. and MacKenzie, A. R.: Polar stratospheric cloud microphysics and chemistry, J. Atmos. Sol.-Terr. Phy., 70, 13-40, https://doi.org/10.1016/j.jastp.2007.09.011, 2008.

Manney, G. L., Santee, M. L., Rex, M., Livesey, N. J., Pitts, M. C., Veefkind, P., Nash, E. R., Wohltmann, I., Lehmann, R., Froidevaux, L., Poole, L. R., Schoeberl, M. R., Haffner, D. P., Davies, J., Dorokhov, V., Gernandt, H., Johnson, B., Kivi, R., Kyro, E., Larsen, N., Levelt, P. F., Makshtas, A., McElroy, C. T., Nakajima, H., Parrondo, M. C., Tarasick, D. W., von der Gathen, P., Walker, K. A., and Zinoviev, N. S.: Unprecedented Arctic ozone loss in 2011, Nature, 478, 469-475, https://doi.org/10.1038/nature10556, 2011.

Marti, J. and Mauersberger, K.: A survey and new measurements of ice vapour pressure at temperatures between 170 and $250 \mathrm{~K}$, Geophys. Res. Lett., 20, 363-366, 1993.

Massie, S. T., Bailey, P. L., Gille, J. C., Lee, E. C., Mergenthaler, J. L., Roche, A. E., Kumer, J. B., Fishbein, E. F., Waters, J. W., and Lahoz, W. A.: Spectral sig- natures of polar stratospheric clouds and sulfate aerosol, J. Atmos. Sci., 51, 3027-3044, https://doi.org/10.1175/15200469(1994)051<3027:SSOPSC>2.0.CO;2, 1994.

Molleker, S., Borrmann, S., Schlager, H., Luo, B., Frey, W., Klingebiel, M., Weigel, R., Ebert, M., Mitev, V., Matthey, R., Woiwode, W., Oelhaf, H., Dörnbrack, A., Stratmann, G., Grooß, J.U., Günther, G., Vogel, B., Müller, R., Krämer, M., Meyer, J., and Cairo, F.: Microphysical properties of synoptic-scale polar stratospheric clouds: in situ measurements of unexpectedly large $\mathrm{HNO}_{3}$-containing particles in the Arctic vortex, Atmos. Chem. Phys., 14, 10785-10801, https://doi.org/10.5194/acp-14-107852014, 2014.

Newman, P. A. and Nash, E. R.: The unusual Southern Hemisphere stratosphere winter of 2002, J. Atmos. Sci., 62, 614-628, https://doi.org/10.1175/JAS-3323.1, 2005.

Orr, A., Hosking, J. S., Hoffmann, L., Keeble, J., Dean, S. M., Roscoe, H. K., Abraham, N. L., Vosper, S., and Braesicke, P.: Inclusion of mountain-wave-induced cooling for the formation of PSCs over the Antarctic Peninsula in a chemistry-climate model, Atmos. Chem. Phys., 15, 1071-1086, https://doi.org/10.5194/acp-15-1071-2015, 2015.

Peter, T. and Grooß, J.-U.: Polar Stratospheric Clouds and Sulfate Aerosol Particles: Microphysics, Denitrification and Heterogeneous chemistry, in: Stratospheric Ozone Depletion and Climate Change, edited by: Rolf Müller, RSC Publishing, 2011, 108-144, 2012.

Pitts, M. C., Poole, L. R., and Thomason, L. W.: CALIPSO polar stratospheric cloud observations: second-generation detection algorithm and composition discrimination, Atmos. Chem. Phys., 9, 7577-7589, https://doi.org/10.5194/acp-9-7577-2009, 2009.

Pitts, M. C., Poole, L. R., Dörnbrack, A., and Thomason, L. W.: The 2009-2010 Arctic polar stratospheric cloud season: a CALIPSO perspective, Atmos. Chem. Phys., 11, 2161-2177, https://doi.org/10.5194/acp-11-2161-2011, 2011.

Pitts, M. C., Poole, L. R., Lambert, A., and Thomason, L. W.: An assessment of CALIOP polar stratospheric cloud composition classification, Atmos. Chem. Phys., 13, 2975-2988, https://doi.org/10.5194/acp-13-2975-2013, 2013.

Pitts, M. C., Poole, L. R., and Gonzalez, R.: Polar stratospheric cloud climatology based on CALIPSO spaceborne lidar measurements from 2006-2017, Atmos. Chem. Phys. Discuss., https://doi.org/10.5194/acp-2018-234, in review, 2018.

Raspollini, P., Carli, B., Carlotti, M., Ceccherini, S., Dehn, A., Dinelli, B. M., Dudhia, A., Flaud, J.-M., López-Puertas, M., Niro, F., Remedios, J. J., Ridolfi, M., Sembhi, H., Sgheri, L., and von Clarmann, T.: Ten years of MIPAS measurements with ESA Level 2 processor V6 - Part 1: Retrieval algorithm and diagnostics of the products, Atmos. Meas. Tech., 6, 2419-2439, https://doi.org/10.5194/amt-6-2419-2013, 2013.

re3data.org: MIPAS/Envisat Observations of Polar Stratospheric Clouds, 2017-09-07 Edn., re3data.org - Registry of Research Data Repositories, https://doi.org/10.17616/R3BN26 last access: 22 September 2017.

Rex, M., Salawitch, R. J., von der Gathen, P., Harris, N. R. P., Chipperfield, M. P., and Naujokat, B.: Arctic ozone loss and climate change, Geophys. Res. Lett., 31, L04116, https://doi.org/10.1029/2003GL018844, 2004. 
Rieder, H. E. and Polvani, L. M.: Are recent Arctic ozone losses caused by increasing greenhouse gases?, Geophys. Res. Lett., 40, 4437-4441, 2013.

Salawitch, R. J., Gobbi, G. P., Wofsy, S. C., and McElroy, M. B.: Denitrification in the Antarctic stratosphere, Nature, 339, 525527, https://doi.org/10.1038/339525a0, 1989.

Sander, S. P., Friedl, R. R., Barker, J. R., Golden, D. M., Kurylo, M. J., Wine, P. H., Abbatt, J. P. D., Burkholder, J. B., Kolb, C. E., Moortgat, G. K., Huie, R. E., and Orkin, V. L.: Chemical kinetics and photochemical data for use in atmospheric studies, JPL Publication 10-6, 2011.

Sembhi, H., Remedios, J., Trent, T., Moore, D. P., Spang, R., Massie, S., and Vernier, J.-P.: MIPAS detection of cloud and aerosol particle occurrence in the UTLS with comparison to HIRDLS and CALIOP, Atmos. Meas. Tech., 5, 2537-2553, https://doi.org/10.5194/amt-5-2537-2012, 2012.

Shi, Q., Jayne, J. T., Kolb, C. E., Worsnop, D. R., and Davidovits, P.: Kinetic model for reaction of $\mathrm{ClONO}_{2}$ with $\mathrm{H}_{2} \mathrm{O}$ and $\mathrm{HCl}$ and $\mathrm{HOCl}$ with $\mathrm{HCl}$ in sulfuric acid solutions, J. Geophys. Res., 106, 24259-24274, https://doi.org/10.1029/2000JD000181, 2001.

Sinnhuber, B. M., Stiller, G., Ruhnke, R., von Clarmann, T., Kellmann, S., and Aschmann, J.: Arctic winter 2010/2011 at the brink of an ozone hole, Geophys. Res. Lett., 38, L24814, https://doi.org/10.1029/2011gl049784, 2011.

Solomon, S.: Stratospheric ozone depletion: a review of concepts and history, Rev. Geophys., 37, 275-316, https://doi.org/10.1029/1999RG900008, 1999.

Solomon, S., Kinnison, D., Bandoro, J., and Garcia, R.: Simulation of polar ozone depletion: An update, J. Geophys. Res.-Atmos., 120, 7958-7974, https://doi.org/10.1002/2015JD023365, 2015.

Spang, R. and Remedios, J.: Observations of a distinctive infra-red spectral feature in the atmospheric spectra of polar stratospheric clouds measured by the CRISTA instrument, Geophys. Res. Lett., 30, 1875, https://doi.org/10.1029/2003GL017231, 2003.

Spang, R., Riese, M., and Offermann, D.: CRISTA-2 observations of the south polar vortex in winter 1997: A new data set for polar process studies, Geophys. Res. Lett., 28, 3159-3162, https://doi.org/10.1029/2000GL012374, 2001.

Spang, R., Remedios, J. J., and Barkley, M.: Colour Indices for the Detection and Differentiation of Cloud Types in Infra-red Limb Emission Spectra, Adv. Space Res., 33, 1041-1047, 2004.

Spang, R., Remedios, J. J., Kramer, L. J., Poole, L. R., Fromm, M. D., Müller, M., Baumgarten, G., and Konopka, P.: Polar stratospheric cloud observations by MIPAS on ENVISAT: detection method, validation and analysis of the northern hemisphere winter 2002/2003, Atmos. Chem. Phys., 5, 679-692, https://doi.org/10.5194/acp-5-679-2005, 2005a.

Spang, R., Remedios, J. J., Tilmes, S., and Riese, M., MIPAS observation of polar stratospheric clouds in the Arctic 2002/2003 and Antarctic 2003 winters, Adv. Space Res., 36, 868-878, 2005 b.

Spang, R., Arndt, K., Dudhia, A., Höpfner, M., Hoffmann, L., Hurley, J., Grainger, R. G., Griessbach, S., Poulsen, C., Remedios, J. J., Riese, M., Sembhi, H., Siddans, R., Waterfall, A., and Zehner, C.: Fast cloud parameter retrievals of MIPAS/Envisat, Atmos. Chem. Phys., 12, 7135-7164, https://doi.org/10.5194/acp-127135-2012, 2012.

Spang, R., Günther, G., Riese, M., Hoffmann, L., Müller, R., and Griessbach, S.: Satellite observations of cirrus clouds in the Northern Hemisphere lowermost stratosphere, Atmos. Chem.
Phys., 15, 927-950, https://doi.org/10.5194/acp-15-927-2015, 2015.

Spang, R., Hoffmann, L., Höpfner, M., Griessbach, S., Müller, R., Pitts, M. C., Orr, A. M. W., and Riese, M.: A multi-wavelength classification method for polar stratospheric cloud types using infrared limb spectra, Atmos. Meas. Tech., 9, 3619-3639, https://doi.org/10.5194/amt-9-3619-2016, 2016.

Strawa, A. W., Drdla, K., Fromm, M., Pueschel, R. F., Hoppel, K. W., Browell, E. V., Hamill, P., and Dempsey, D. P.: Discriminating Types Ia and Ib polar stratospheric clouds in POAM satellite data, J. Geophys. Res., 107, 8291, https://doi.org/10.1029/2001JD000458, 2002.

Tabazadeh, A., Jensen, E. J., Toon, O. B., Drdla, K., and Schoeberl, M. R.: Role of the Stratospheric Polar Freezing Belt in Denitrification, Science, 291, 2591-2594, 2001.

Taylor, F. W., Lambert, A., Grainger, R. G., Rodgers, C. D., and Remedios, J. J.: Properties of Northern Hemisphere Polar Stratospheric Clouds and Volcanic Aerosol in 1991/2 from UARS/ISAMS Satellite Measurements, J. Atmos. Sci., 51, 30193026, 1994.

Tilmes, S., Müller, R., Grooß, J.-U., and Russell III, J. M.: Ozone loss and chlorine activation in the Arctic winters 1991-2003 derived with the tracer-tracer correlations, Atmos. Chem. Phys., 4, 2181-2213, https://doi.org/10.5194/acp-4-2181-2004, 2004.

Tritscher, I., Grooß, J.-U., Spang, R., Pitts, M. C., Müller, R., and Riese, M.: Lagrangian simulation of ice particles and resulting dehydration in the polar winter stratosphere, Atmos. Chem. Phys. Discuss., in preparation, 2018.

Vanhellemont, F., Fussen, D., Mateshvili, N., Tétard, C., Bingen, C., Dekemper, E., Loodts, N., Kyrölä, E., Sofieva, V., Tamminen, J., Hauchecorne, A., Bertaux, J.-L., Dalaudier, F., Blanot, L., Fanton d'Andon, O., Barrot, G., Guirlet, M., Fehr, T., and Saavedra, L.: Optical extinction by upper tropospheric/stratospheric aerosols and clouds: GOMOS observations for the period 2002-2008, Atmos. Chem. Phys., 10, 7997-8009, https://doi.org/10.5194/acp10-7997-2010, 2010.

von Hobe, M., Bekki, S., Borrmann, S., Cairo, F., D’Amato, F., Di Donfrancesco, G., Dörnbrack, A., Ebersoldt, A., Ebert, M., Emde, C., Engel, I., Ern, M., Frey, W., Genco, S., Griessbach, S., Grooß, J.-U., Gulde, T., Günther, G., Hösen, E., Hoffmann, L., Homonnai, V., Hoyle, C. R., Isaksen, I. S. A., Jackson, D. R., Jánosi, I. M., Jones, R. L., Kandler, K., Kalicinsky, C., Keil, A., Khaykin, S. M., Khosrawi, F., Kivi, R., Kuttippurath, J., Laube, J. C., Lefèvre, F., Lehmann, R., Ludmann, S., Luo, B. P., Marchand, M., Meyer, J., Mitev, V., Molleker, S., Müller, R., Oelhaf, H., Olschewski, F., Orsolini, Y., Peter, T., Pfeilsticker, K., Piesch, C., Pitts, M. C., Poole, L. R., Pope, F. D., Ravegnani, F., Rex, M., Riese, M., Röckmann, T., Rognerud, B., Roiger, A., Rolf, C., Santee, M. L., Scheibe, M., Schiller, C., Schlager, H., Siciliani de Cumis, M., Sitnikov, N., Søvde, O. A., Spang, R., Spelten, N., Stordal, F., Suminska-Ebersoldt, O., Ulanovski, A., Ungermann, J., Viciani, S., Volk, C. M., vom Scheidt, M., von der Gathen, P., Walker, K., Wegner, T., Weigel, R., Weinbruch, S., Wetzel, G., Wienhold, F. G., Wohltmann, I., Woiwode, W., Young, I. A. K., Yushkov, V., Zobrist, B., and Stroh, F.: Reconciliation of essential process parameters for an enhanced predictability of Arctic stratospheric ozone loss and its climate interactions (RECONCILE): activities and results, Atmos. Chem. Phys., 13, 92339268, https://doi.org/10.5194/acp-13-9233-2013, 2013. 
von Savigny, C., Ulasi, E. P., Eichmann, K.-U., Bovensmann, H., and Burrows, J. P.: Detection and mapping of polar stratospheric clouds using limb scattering observations, Atmos. Chem. Phys., 5, 3071-3079, https://doi.org/10.5194/acp-5-3071-2005, 2005.

Waibel, A. E., Peter, T., Carslaw, K. S., Oelhaf, H., Wetzel, G., Crutzen, P. J., Pöschl, U., Tsias, A., Reimer, E., and Fischer, H.: Arctic Ozone Loss Due to Denitrification, Science, 283, 2064 2069, https://doi.org/10.1126/science.283.5410.2064, 1999.

Wegner, T., Grooß, J.-U., von Hobe, M., Stroh, F., SuminskaEbersoldt, O., Volk, C. M., Hösen, E., Mitev, V., Shur, G., and Müller, R.: Heterogeneous chlorine activation on stratospheric aerosols and clouds in the Arctic polar vortex, Atmos. Chem. Phys., 12, 11095-11106, https://doi.org/10.5194/acp-12-110952012, 2012.

Wegner, T., Pitts, M. C., Poole, L. R., Tritscher, I., Grooß, J.U., and Nakajima, H.: Vortex-wide chlorine activation by a mesoscale PSC event in the Arctic winter of 2009/10, Atmos. Chem. Phys., 16, 4569-4577, https://doi.org/10.5194/acp-164569-2016, 2016.

Winker, D. M., Vaughan, M. A., Omar, A., Hu, Y., Powell, K. A., Liu, Z., Hunt, W. H., and Young, S. A.: Overview of the CALIPSO Mission and CALIOP Data Processing Algorithms, J. Atmos. Ocean. Tech., 26, 2310-2323, https://doi.org/10.1175/2009JTECHA1281.1, 2009.
WMO (World Meteorological Organization): Scientific Assessment of Ozone Depletion 2014, World Meteorological Organization, Global Ozone Research and Monitoring Project-Report No. 55, 416 pp., Geneva, Switzerland, 2014.

Wohltmann, I., Wegner, T., Müller, R., Lehmann, R., Rex, M., Manney, G. L., Santee, M. L., Bernath, P., Suminska-Ebersoldt, O., Stroh, F., von Hobe, M., Volk, C. M., Hösen, E., Ravegnani, F., Ulanovsky, A., and Yushkov, V.: Uncertainties in modelling heterogeneous chemistry and Arctic ozone depletion in the winter 2009/2010, Atmos. Chem. Phys., 13, 3909-3929, https://doi.org/10.5194/acp-13-3909-2013, 2013.

Wu, D. L. and Jiang, J. H.: MLS observations of atmospheric gravity waves over Antarctica, J. Geophys. Res., 107, 4773, https://doi.org/10.1029/2002JD002390, 2002.

Wu, X., Griessbach, S., and Hoffmann, L.: Equatorward dispersion of a high-latitude volcanic plume and its relation to the Asian summer monsoon: a case study of the Sarychev eruption in 2009, Atmos. Chem. Phys., 17, 13439-13455, https://doi.org/10.5194/acp-17-13439-2017, 2017. 